MPP-2005-41

HU-EP-05/21

hep-th/0505071

\title{
Wrapping interactions and the genus expansion of the 2-point function of composite operators
}

\author{
Christoph Sieg ${ }^{1}$ and Alessandro Torrielli ${ }^{2}$ \\ 11) Max-Planck-Institut für Physik \\ Föhringer Ring 6, D-80805 München \\ 2) Humboldt-Universität zu Berlin, Institut für Physik \\ Newtonstraße 15, D-12489 Berlin
}

\begin{abstract}
We perform a systematic analysis of wrapping interactions for a general class of theories with color degrees of freedom, including $\mathcal{N}=4$ SYM. Wrapping interactions arise in the genus expansion of the 2-point function of composite operators as finite size effects that start to appear at a certain order in the coupling constant at which the range of the interaction is equal to the length of the operators. We analyze in detail the relevant genus expansions, and introduce a strategy to single out the wrapping contributions, based on adding spectator fields. We use a toy model to demonstrate our procedure, performing all computations explicitly. Although completely general, our treatment should be particularly useful for applications to the recent problem of wrapping contributions in some checks of the AdS/CFT correspondence.
\end{abstract}

\footnotetext{
1 csieg@mppmu.mpg.de

2 torriell@physik.hu-berlin.de
} 


\section{Contents}

1 Introduction 2

2 The 2-point function of composite operators 5

2.1 Building blocks ..................... 5

2.2 Coupling and genus expansion of $V_{2 R} \ldots \ldots . . . . . .66$

2.3 Coupling and genus expansion of the 2-point function . . . . . . 7

$\begin{array}{lll}3 & \text { Wrapping diagrams } & 11\end{array}$

3.1 Definition of wrapping and non-wrapping diagrams . . . . . . 11

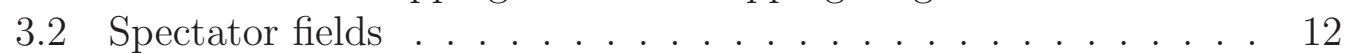

3.3 The encoding of the topologv of planar wrapping diagrams by the spectator fields 14

4 Planar contributions to the 2-point function $\quad 19$

4.1 Non-interacting case . . . . . . . . . . . . . . . . 19

4.2 Generic planar connected contributions to $V_{2 D}$. . . . . . . . . . 20

4.3 Generic planar wrapping contributions to $V_{2 B} \ldots \ldots . . .21$

4.4 Generic planar wrapping contributions to $V_{2 B}$ including flavor . 24

5 The tov model 25

5.14 -vertex with cyclic svmmetric flavor flux . . . . . . . . . . . . . 28

5.24 -vertex with commutators . . . . . . . . . . . . . . 30

6 A candidate for $\downarrow$

\begin{tabular}{|ll|}
\hline 7 & Conclusions \\
\hline
\end{tabular}

\begin{tabular}{|l|}
\hline Acknowledgements \\
\hline 5
\end{tabular}

$\begin{array}{ll}\text { A Counting rules for Feynman diagrams } & 36\end{array}$

B Rules for $U(N)$ and $S U(N)$

\begin{tabular}{ll}
\hline Bibliography & 39
\end{tabular} 


\section{Introduction}

The AdS/CFT correspondence [1] claims that type II B string theory on an $\mathrm{AdS}_{5} \times \mathrm{S}^{5}$ background with Ramond-Ramond (RR) flux is dual to $\mathcal{N}=4$ super Yang-Mills (SYM) theory with gauge group $S U(N)$ which is invariant under the superconformal symmetry group $S U(2,2 \mid 4)$. The common curvature radius $R$ of $\mathrm{AdS}_{5}$ and of $\mathrm{S}^{5}$ in units of the string length $\sqrt{\alpha^{\prime}}$ is related to the 't Hooft coupling $\lambda$ via

$$
\frac{R^{2}}{\alpha^{\prime}}=\sqrt{\lambda}, \quad \lambda=g^{2} N, \quad g^{2}=4 \pi g_{\mathrm{s}},
$$

where $g$ and $g_{\mathrm{s}}$ are the SYM and string coupling constants, respectively. One important motivation for this conjecture is that the isometry group $S O(2,4) \times$ $S O(6)$ of $\mathrm{AdS}_{5} \times \mathrm{S}^{5}$ can be identified with the bosonic subgroup $S O(2,4) \times$ $S U(4)$ of the superconformal group. A general proof of the conjecture is still out of reach, mainly due to the fact that a quantization of string theories in backgrounds with RR flux is difficult. Furthermore, the duality relates the strong coupling regime of one theory to the weak coupling regime of the other theory, preventing one from directly using perturbation theory to compute results in both theories that can be compared in a common regime of the parameters.

However, it is possible to probe the correspondence in different limits. In one of these limits one considers classical solutions on the string side 2, 3] (see also the review [4 and references therein). In this case quantitative results can be computed in the string theory as well as in its dual gauge theory. In the regime of large quantum numbers of the string (here collectively denoted by the angular momentum $J$ of a rotating string solution) and in the large tension limit $(\sqrt{\lambda} \gg 1)$ the classical energy has a regular expansion in the modified 't Hooft coupling $\lambda^{\prime}=\frac{\lambda}{J^{2}}$ which is held fixed [5]. The analysis can be extended by including quantum fluctuations around the classical string solution [3, 5]. The classical string sector shows integrability, e.g. rotating (rigid) string solutions have been shown to be described by the classical integrable Neumann system [6]. See also [7] for a generalization and [8, 9, 10, 11, 12] for related work on integrability in this context.

The AdS/CFT correspondence predicts a matching of the classical energy of closed strings with the eigenvalues of the anomalous dimension matrix that describes the mixing of composite operators of $\mathcal{N}=4 \mathrm{SYM}$ under renormalization. These operators contain a single trace over the gauge group and can 
be regarded as discretized closed strings. In particular, the limit of interest requires that the operators contain a huge number of fields, implying that their mixing matrix, that has to be diagonalized, contains a huge number of entries. The mixing matrix itself can be obtained from the 2-point functions of the composite operators. Alternatively, the mixing problem can be recast in terms of an eigenvalue problem for the dilatation operator of $\mathcal{N}=4$ SYM [13] (see also the reviews [14, 15] and references therein). Furthermore, in the planar limit, the mixing problem has been reformulated in terms of integrable spin chains [16]. There, the composite single-trace operators are regarded as cyclic spin chains. Each fundamental field 'flavor' within the trace is interpreted as a spin projection eigenvalue at the corresponding site of the chain. The dilatation operator itself becomes the Hamiltonian of the chain. Formulated in these terms, the Bethe ansatz [17] (see [18] for a review) provides a tool for finding the energy eigenvalues for the spin chain states and thus the eigenvalues of the anomalous dimension matrix.

Remarkable agreement of the classical string energies with the anomalous dimensions is found up to two loops. However, a mismatch was observed at three loops [19]. Another mismatch has been observed in the BMN limit [20] of the AdS/CFT correspondence. This limit corresponds to an expansion around a pointlike classical string in the center of $\mathrm{AdS}_{5}$ that moves along a great circle in $S^{5}$. In this case, moving with the pointlike string with the velocity of light, the $\mathrm{AdS}_{5} \times \mathrm{S}^{5}$ background of the AdS/CFT correspondence is transformed via the Penrose-Güven limit [21, 22] to the 10-dimensional plane wave background [23. 24]. The first order quantum fluctuations around the pointlike solutions are then described by strings in this plane wave background 3]. The corrections to the energy caused by higher order quantum fluctuations, i.e. curvature corrections to the plane wave background [25, show a mismatch with the corresponding anomalous dimensions starting at three loops [26, 27, 28]. There have been first indications for a mismatch between the quantum fluctuations at one loop order of extended string solutions, rotating in $S^{5}[5]$ and in both, $\operatorname{AdS}_{5}$ and $S^{5}$ [29], with the respective gauge theory results [30, 31]. However, agreement at this order was found later by including an overlooked contribution to the Bethe ansatz 32. ${ }^{3}$

A possible explanation for the mismatch, that does not insist on the unsatisfactory possibility of a breakdown of the AdS/CFT correspondence and of integrability in the planar limit, might be a non-commutativity of the lim-

\footnotetext{
${ }^{3}$ We would like to thank I. Y. Park for bringing this fact to our attention.
} 
its taken on both sides [33, 34. On the string side, $J \rightarrow \infty$ is taken first, and then the expression for the energy is expanded in powers of $\lambda^{\prime}$. However, on the gauge side the anomalous dimensions are computed as a perturbation expansion in $\lambda$ that is valid for sufficiently large but finite $J$, and then the limit $J \rightarrow \infty$ is taken, keeping $\lambda^{\prime}$ fixed. Thereby, one has ignored finite size effects, that become relevant whenever the spin chain length is less than the range of the interaction. If this is the case, there are contributions in which the interaction wraps around the state [33, 34. They are denoted as wrapping interactions. First quantitative results including these interactions have been obtained in the context of a computer-based study of the plane wave matrix model at four loop order 35 .

The purpose of this paper is to present a systematic and general study of wrapping interactions, not relying on the issues of a concrete theory and on a given perturbative order in the coupling constant. Instead, it contains statements valid in a class of theories which includes $\mathcal{N}=4 \mathrm{SYM}$. Our general results are then checked at low and fixed loop order with the help of a simple example, that is then refined in subsequent steps. In this way we hope to have provided an initial step to understand the role of the wrapping interactions in the concrete challenge of explaining the above mentioned mismatch between semiclassical string energies and anomalous dimensions.

The paper is organized as follows:

In Section 2 we introduce and discuss the 2-point function of composite operators, being generated by connecting the legs of the operators with a Green function of the theory. We then work out the differences of the genus expansion of such a Green function and of the obtained 2-point function.

In Section 3 we define and classify wrapping interactions as particular contributions to the Green functions that contribute at lower genus to the 2-point function. We work out some general statements for wrapping interactions by adding spectator fields to the composite operators. A special case of particular interest in the context of the AdS/CFT correspondence is the case of planar contributions to the 2-point function. There, we find that the planar wrapping diagrams originate from genus one contribution to the Green functions. We identify a unique structure for the spectator fields of planar wrapping diagrams.

In Section 4 we present the expressions for the effective vertices and show their spectator structures.

In Section 5 we apply our results to a toy model, given by scalar $\phi^{4}$ theory with a massless colored field of a single flavor. The toy model is then extended 
to multiple flavors and interactions with different flavor fluxes. At the end we arrive at the 4 -scalar interaction of $\mathcal{N}=4 \mathrm{SYM}$.

In Section [ 6 discuss a possibility to choose the spectator fields in such a way that wrapping interactions can be automatically projected out in computerbased symbolic computations.

In Appendix $\mathrm{A}$ we review and summarize some counting rules for Feynman diagrams used in the main text. We then apply them to extract some issues of (planar) wrapping diagrams.

In Appendix B we collect some useful formulae for $S U(N)$ and $U(N)$ which enter our calculations.

\section{The 2-point function of composite operators}

\subsection{Building blocks}

Before we can define wrapping interactions we should fix our notations and conventions when we deal with the 2-point functions of composite operators.

By $\varnothing e R, e=1,2$ we denote two local operators that consist of $r=1, \ldots, R$ elementary fields $\phi_{n_{r}}$ where each $n_{r}$ denotes one field 'flavor' of the theory. Of particular interest are single-trace operators of the form

$$
\emptyset R=\frac{1}{\sqrt{N}^{R-2}} \operatorname{tr}\left(\phi_{n_{1}} \ldots \phi_{n_{R}}\right),
$$

where the trace runs over the gauge group, i.e. the elementary fields $\phi_{n_{r}}$ are decomposed as $\phi_{n_{r}}=\phi_{n_{r}}^{a} T^{a}$, and $T^{a}$ are $N \times N$ representation matrices of the Lie algebra of the gauge group. Later we will refer to the chosen normalization factor.

For the sake of simplicity, from now on we will use the abbreviation

$$
\left(a_{1} a_{2} \ldots a_{n}\right)=\operatorname{tr}\left(T^{a_{1}} T^{a_{2}} \ldots T^{a_{n}}\right),
$$

for the traces. We represent $\varnothing R$ by a rectangular box with $R$ elementary external legs attached to one of its sides. Counting of the lines starts with that line which with view into the outgoing direction has no direct neighbour on its right hand side, and it ends with the line that has no left hand neighbour, see Fig. 1(a).

By $V_{2 R}$ we denote the Green functions of the theory with $2 R$ elementary external legs. $R$ of them will be regarded as ingoing and outgoing, respectively. 


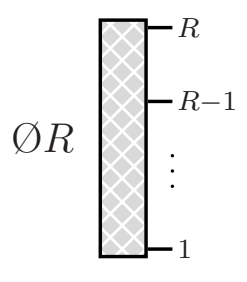

(a)

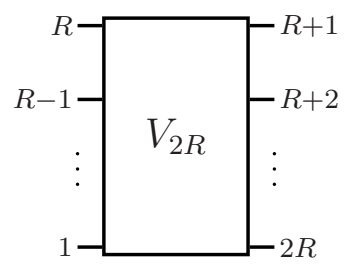

(b)

Figure 1: The composite operators $\varnothing R(\mathrm{a})$ and the Green function $V_{2 R}$ (b)] are the building blocks of the 2-point function $\left(\varnothing 1 R, V_{2 R}, \varnothing 2 R\right)$

We represent $V_{2 R}$ as a rectangular box where $R$ elementary field lines enter from the left hand side and the $R$ remaining lines from the right hand side. Counting of the lines is clockwise and starts from the lower left corner, see Fig. 1(b)

The 2-point function of two composite operators $\varnothing e R$, is defined as the correlation function in which the $R$ legs of the operator $\varnothing 1 R[\varnothing 2 R]$ are entirely contracted with the $R$ ingoing [outgoing] fields of $V_{2 R}$. We denote such a 2point function by $\left(\varnothing 1 R, V_{2 R}, \varnothing 2 R\right)$. When the two $\varnothing e R$ and $V_{2 R}$ are combined within a 2-point function, the elementary fields within the traces of $\varnothing e R$ are counted with increasing labels from left to right, starting with $\varnothing 1 R$ from 1 to $R$, and continuing with $\varnothing 2 R$ using the labels $R+1$ to $2 R$. The 2 -point function is obtained by contracting the $2 R$ fields of the operators with the fields of $V_{2 R}$ that carry the same label. This procedure guarantees that there are no crossings between the connecting lines if the lines leave the operator on the left [right] hand side from its right [left] hand side.

\subsection{Coupling and genus expansion of $V_{2 R}$}

In perturbation theory, the Green function $V_{2 R}$ can be regarded as a series in powers $K$ of the coupling constant $g$. Each fixed power $g^{K}$ contains a number

of elementary Feynman diagrams. Denoting a particular diagram by $D_{2 R}^{K}$, one 
can write

$$
V_{2 R}=\sum_{K} \sum_{D} D_{2 R}^{K}
$$

In this expansion, any diagram $D_{2 R}^{K}$ is of the order $g^{K}$. Furthermore, one can perform a genus expansion in powers of $N^{-2}$ [36]. Each Feynman diagram $D_{2 R}^{K}$ is itself a sum of diagrams $D_{2 R}^{K(h)}$ which differ in the genus $h$. One defines the genus $h$ of a diagram as the minimal genus of all compact Riemannian surfaces on which the diagram can be drawn in double-line notation without any crossings of lines. On the compactified Riemann surface, the $2 R$ external lines end at a common vertex (at the point representing $\infty$ ) with the reversed ordering, i.e. they are attached counter-clockwise, starting with the lowest label. This configuration is shown in Fig. 2. Its genus can be obtained by Euler's relation for the Euler character $\chi$

$$
\chi=2-2 h=V-P+I,
$$

where $V$ is the number of vertices, $P$ is the number of propagators, and $I$ is the number of index loops.

The expansion of the Green function $V_{2 R}$ then reads

$$
V_{2 R}=\sum_{h} V_{2 R}^{(h)}=\sum_{h} \sum_{K} \sum_{D} D_{2 R}^{K(h)}
$$

where each contribution $V_{2 R}^{(h)}$ with fixed genus $h$ is of order $N^{2-2 h}$, and each diagram $D_{2 R}^{K(h)}$ is of the order $\sqrt{\lambda}^{K} N^{2-2 h}$.

The vertex at $\infty$ carries a normalization factor $N^{1-R}$, which can be understood as follows. The vertex at $\infty$ can be regarded as an effective vertex for a planar connected $(C=1)$ tree-level $(L=0)$ diagram with $E=2 R$ external legs. According to A.3 it can be built out of $2 R-23$-vertices. Hence, it

should depend on $\sqrt{\lambda}^{2 R-2}$. The normalization factor is obtained by providing the required $N$ dependence to transform the YM coupling $g$ into $\lambda$, and then setting $\lambda=1$ for the effective vertex.

\subsection{Coupling and genus expansion of the 2-point func- tion}

Similarly to $V_{2 R}$, one can expand the 2-point function $\left(\varnothing 1 R, V_{2 R}, \varnothing 2 R\right)$ in powers of $g$ and $N^{-2}$. A single diagram $D_{2 R}^{K(h)}$ generates a single diagram of 


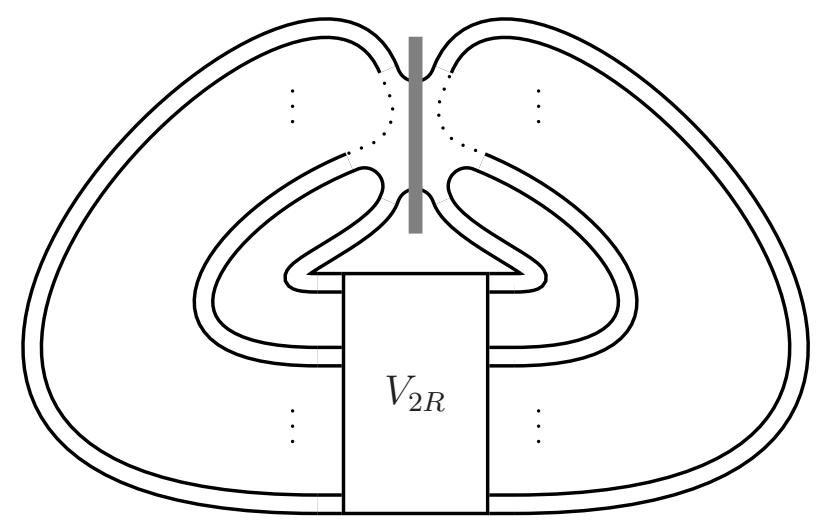

Figure 2: On a compact Riemann surface, the external lines of the Green function $V_{2 R}$ are connected to the vertex at $\infty$. These connections separate the Riemann suface into two parts. Lines that cross these connections require adding of the wrapping handle, depicted in gray.

the 2-point function if all $(R !)^{2}$ permutations within the two bundles of $R$ elementary legs are contained within $V_{2 R}$. This means each permutation of the external legs is regarded as a different diagram $D_{2 R}^{K(h)}$. Then, one only has to consider a unique way to contract the $2 R$ legs of the two operators $\varnothing e R$ with the external legs of $D_{2 R}^{K(h)}$.

Consider the genus expansion of the 2-point function $\left(\varnothing 1 R, V_{2 R}, \varnothing 2 R\right)$. By $\left(\varnothing 1 R, D_{2 R}^{K(h)}, \varnothing 2 R\right)^{(H)}$ we denote the genus $H$ contribution of a particular diagram $D_{2 R}^{K(h)}$ to the 2-point function. The genus $h$ of a particular diagram $D_{2 R}^{K(h)}$ does not uniquely determine the genus $H$. This effect has two reasons.

First of all, drawing a particular diagram of the 2-point function, one has drawn only one representative of an $R^{2}$-dimensional equivalence class of diagrams. It contains all diagrams that differ only by cyclic permutations within each of the two pairs of legs that connect the diagram $D_{2 R}^{K(h)}$ to the two operators $\varnothing e 2 R$. The equivalence relation is based on the fact that the operators (2.1) are invariant under cyclic permutations of their elementary fields. Two different representations contain two different diagrams $D_{2 R}^{K(h)}$ and $D_{2 R}^{K\left(h^{\prime}\right)}$ that can be mapped to each other by acting with cyclic permutations on the ingoing and/or outgoing legs. All these diagrams are again members of an equivalence 


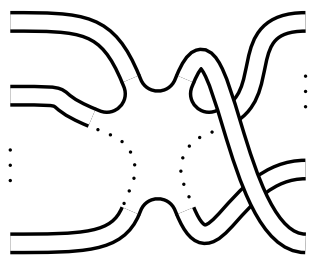

(a)

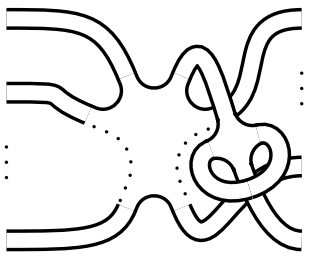

(b)

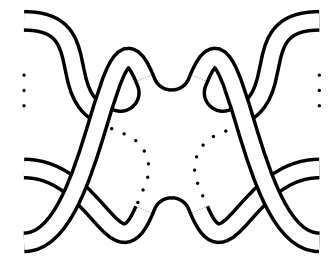

(c)

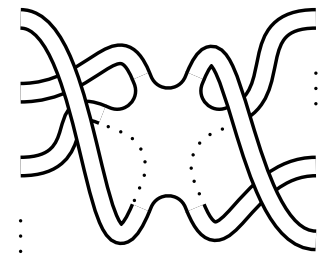

(d)

Figure 3: The cyclic permutations of the external legs have been moved to the vertex at $\infty$. The resolution of the cyclic permutation requires adding one handle (a). An exception is the case (b) in which the crossing leg contains a non-planar self energy correction. In this case the genus does not change. Two cyclic permutations in the same (c) or in opposite directions (d) can be resolved with a single handle.

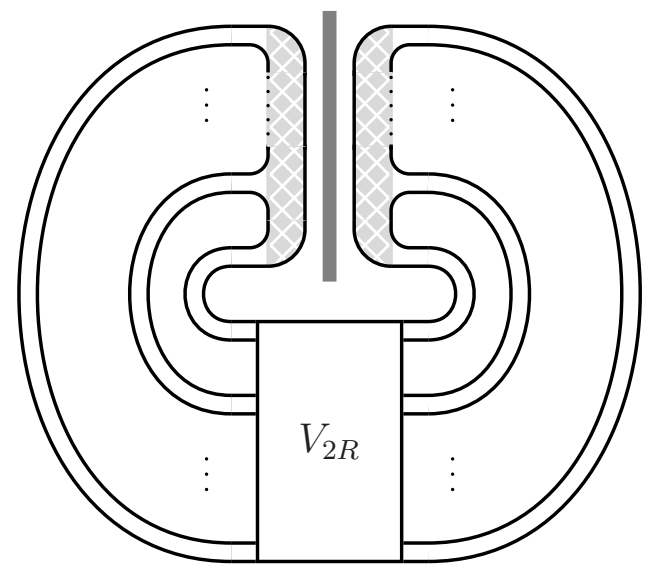

Figure 4: On a compact Riemann surface, the two operators $\varnothing e R$ in the 2-point function have a finite separation. This allows one to occupy the wrapping path, depicted in gray. 
class, for which elements one has $h^{\prime} \neq h$ in general. Let us denote by $h$ the minimal genus of all the diagrams within one equivalence class. A cyclic permutation applied to either the incoming or outgoing legs increases the genus $h$ by at most one. This can be seen by resolving the crossing at the vertex at $\infty$, see Fig. 3(a), It requires adding at most one handle. In case that the crossing line contains a non-planar self energy contribution, no futher handle has to be added. A further cyclic permutation acting on the remaining $R$ legs does not change the genus further if the cyclic permutation applied to the other $R$ legs has already increased the genus. This is because the same handle can be used to resolve the crossing, see Fig. $3(\mathrm{c})$ and $3(\mathrm{~d})$. However, in case that no handle had to be added for the first set of $R$ legs (like in case of a crossing line with a non-planar self energy contribution), at most one handle has to be added for the cyclic permutation applied to the second set of $R$ fields.

Under the $R^{2}$ diagrams $D_{2 R}^{K\left(h^{\prime}\right)}$ there is at least one with minimal genus $h^{\prime}=h$. The remaining ones have genus $h \leq h^{\prime} \leq h+1$ because the same handle can be used to resolve the two crossings caused by both cyclic permutations. This effect means that in $\left(\varnothing 1 R, D_{2 R}^{K(h)}, \varnothing 2 R\right)^{(H)}$ the genus $H$ can have values $H=h-1$ or $H=h$.

The second reason for the genus expansion of $V_{2 R}$ and of $\left(\varnothing 1 R, V_{2 R}, \varnothing 2 R\right)$ to differ from each other is caused by the fact that the 2-point function does not have external lines which, when it is drawn on a compact Riemann surface, have to be connected to an additional vertex at $\infty$. The bundles of ingoing and outgoing lines, interacting with each other in $V_{2 R}$ and in the vertex at $\infty$, form a closed contour which divides the Riemann surface into two parts, see Fig. 2. That means, there has to be one handle for all the field lines crossing this contour. We will call this handle the wrapping handle. While being needed in certain diagrams $D_{2 R}^{K(h)}$, it can be removed in $\left(\varnothing 1 R, D_{2 R}^{K(h)}, \varnothing 2 R\right)$. Due to the separation of the two operators $\varnothing e R$ their contraction with the external lines of $V_{2 R}$ no longer forms a closed contour, that divides the Riemann surface into two parts. There is a direct connection, from now on called the wrapping path, that replaces the wrapping handle (compare Fig. 2 and Fig. 4). The genus $H$ of $\left(\varnothing 1 R, D_{2 R}^{K(h)}, \varnothing 2 R\right)$ hence obeys $h-1 \leq H \leq h$. Combining the above described effects, one expects that the diagrams $\left(\varnothing 1 R, D_{2 R}^{K(h)}, \varnothing 2 R\right)^{(H)}$ obtained from a genus $h$ diagram $D_{2 R}^{K(h)}$ in general have $h-2 \leq H \leq h$.

Since here we are interested only in the change of the genus caused by removing the wrapping handle, we will disentangle both effect. From each equivalence class of diagrams we will only use one representative $D_{2 R}^{K(h)}$ with 


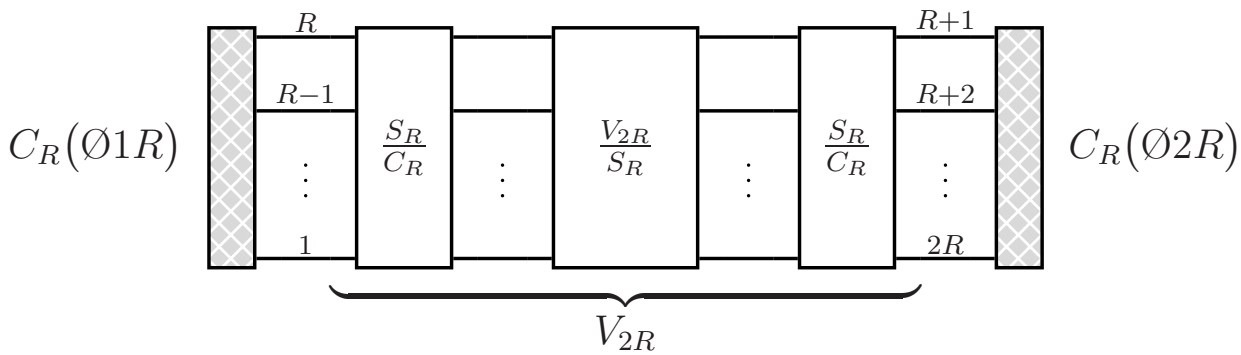

Figure 5: Decomposition of the 2-point function $\left(\varnothing 1 R, V_{2 R}, \varnothing 2 R\right)$ into interaction part $\frac{V_{2 R}}{S_{R}}$ without any permutations of the external legs and the permutations $\frac{S_{R}}{C_{R}}$ without cyclic permutations $C_{R}$. $V_{2 R}$ contains only one diagram $D_{2 R}^{K(h)}$ of each equivalence class with minimal genus $h$. The cyclic permutations are taken into account by using all cyclic permutations of the operators $\varnothing e R$, denoted by $C_{R}(\varnothing e R)$.

the minimal genus $h$ to build the corresponding diagram of the 2-point function. The additonal $R^{2}-1$ diagrams generated by cyclic permutations are then taken care of by the two operators $\varnothing e R$. That means, to each of the operators we associate $R-1$ copies that differ by cyclic permutations. They are combined in $R^{2}$ possible ways to obtain all contributions to the 2 -point function that contain the diagrams $D_{2 R}^{K\left(h^{\prime}\right)}$ with $h \leq h^{\prime} \leq h+1$ in the equivalence class of $D_{2 R}^{K(h)}$. The distribution of the permutations of the external legs of $V_{2 R}$ is depicted in Fig. 5

After that we are left with two types of genus $h$ diagrams $D_{2 R}^{K(h)}$. One type of $D_{2 R}^{K(h)}$ contributes to genus $h$ diagrams of the 2-point function $\left(\varnothing 1 R, D_{2 R}^{K(h)}, \varnothing 2 R\right)$, the other type contributes to genus $h-1$ diagrams, and its elements are called wrapping diagrams. In the next Section we will analyze these contributions in detail.

\section{$3 \quad$ Wrapping diagrams}

\subsection{Definition of wrapping and non-wrapping diagrams}

We define the genus $h-1$ wrapping diagrams $D_{\mathrm{w}, 2 R}^{K(h)}$ as the genus $h$ contributions to $V_{2 R}$ that lead to a genus $H=h-1$ contribution to the 2-point function $\left(\varnothing 1 R, V_{2 R}, \varnothing 2 R\right)$. Correspondingly, the non-wrapping diagrams $D_{\mathrm{nw}, 2 R}^{K(h)}$ 


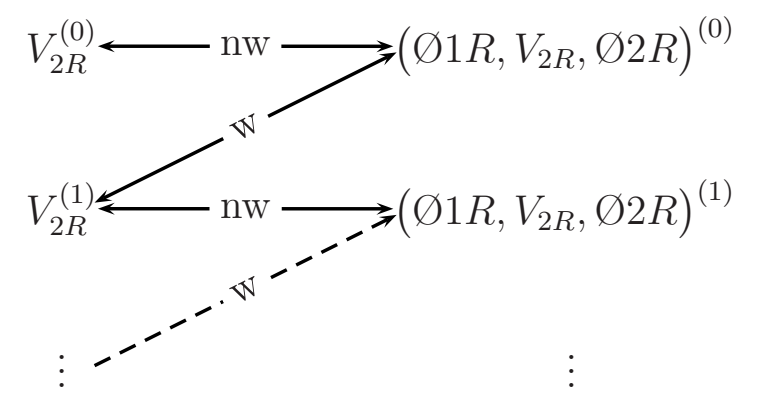

Figure 6: The translation of the genus expansion between $V_{2 R}^{(h)}$ and $\left(\varnothing 1 R, V_{2 R}, \varnothing 2 R\right)^{(H)}$. The non wrapping (nw) genus $h$ contributions $V_{\mathrm{nw}, 2 R}^{(h)}$ to the Green function become genus $H=h$ contributions $\left(\varnothing 1 R, V_{2 R}, \varnothing 2 R\right)^{(H)}$ to the 2-point function. The wrapping (w) contributions $V_{\mathrm{w}, 2 R}^{(h)}$ become genus $H=h-1$ contributions to the 2-point function.

are the remaining genus $h$ diagrams that generate genus $H=h$ contributions to $\left(\varnothing 1 R, V_{2 R}, \varnothing 2 R\right)$. One has the relations

$$
\begin{gathered}
V_{2 R}^{(h)}=V_{\mathrm{nw}, 2 R}^{(h)}+V_{\mathrm{w}, 2 R}^{(h)} \\
\left(\varnothing 1 R, V_{2 R}^{(h)}, \varnothing 2 R\right)=\left(\varnothing 1 R, V_{\mathrm{nw}, 2 R}^{(h)}, \varnothing 2 R\right)^{(h)}+\left(\varnothing 1 R, V_{\mathrm{w}, 2 R}^{(h)}, \varnothing 2 R\right)^{(h-1)}, \\
\left(\varnothing 1 R, V_{2 R}, \varnothing 2 R\right)^{(H)}=\left(\varnothing 1 R, V_{\mathrm{nw}, 2 R}^{(H)}, \varnothing 2 R\right)+\left(\varnothing 1 R, V_{\mathrm{w}, 2 R}^{(H+1)}, \varnothing 2 R\right) .
\end{gathered}
$$

The translation of the genus expansion between $V_{2 R}^{(h)}$ and $\left(\varnothing 1 R, V_{2 R}, \varnothing 2 R\right)^{(H)}$ is visualized in Fig. 6 ,

\subsection{Spectator fields}

Here we develop a useful tool to distinguish between planar wrapping and non wrapping diagrams based on adding spectator fields. They come in pairs of two fields $\psi, \hat{\psi}$ that are inserted into the trace of respectively $\varnothing 12 R$ and $\varnothing 22 R$, defined in (2.1), at any position between two $\phi_{n_{r}}$. The contraction of a pair of 
spectator fields, denoted by $\psi \hat{\psi}$, generates a further connection line within the diagram, not interacting with any other lines. Pairs of spectator fields must be added such that their connection lines do not cross each other, and such that not two pairs are equivalent. Two pairs are equivalent if both, the two $\psi$ and the $\hat{\psi}$ are direct neighbours within the two traces, not being separated by at least one $\phi_{n_{r}}$. The spectator pairs can be used to test some aspects of the topology of a diagram by observing how the genus $H$ of the 2-point function behaves when their connection lines are added.

In contrast to the wrapping diagrams, for non-wrapping ones there exists at least one representation in which the wrapping path introduced in Subsection 2.3 is unoccupied. Hence, following this empty path, it is possible to add a pair of spectator fields such that their connection does not cross any of the other lines.

Due to the fact that the lines along the wrapping path differ between two representations of the same diagram, it is not sufficient for a diagram to be wrapping that its wrapping path is occupied by at least one field line. Likewise, it is not necessary for a diagram to be non-wrapping that its wrapping path is unoccupied. Instead, to distinguish between a wrapping and a non-wrapping diagram, one has to ensure that none or respectively at least one equivalent diagram with an unoccupied wrapping path exists.

Consider a candidate for a genus $h-1$ wrapping diagram. In a given representation of such a diagram one can always free the wrapping path by removing some lines. No further lines that have no influence on the occupation of the wrapping path are removed. In a second step one removes the two operators $\varnothing e 2 R$. In this way one arrives at a non-wrapping diagram $D_{\mathrm{nw}, 2 R}^{K^{\prime}\left(h^{\prime}\right)}$ that is a truncated version of the original diagram $D_{2 R}^{K(h)}$, and that obeys $K^{\prime} \leq K$, $h^{\prime} \leq h-1$. If the original diagram $D_{2 R}^{K(h)}$ was wrapping, then the truncated diagram $D_{\mathrm{nw}, 2 R}^{K^{\prime}\left(h^{\prime}\right)}$ is necessarily connected $(C=1$ in the notation introduced in Appendix A, see (A.1)). This statement is only true if one forbids that $D_{2 R}^{K(h)}$ contains connected pieces that are entirely attached to only one of the two sets of $R$ external legs. We show that if $C>1$ under the above assumptions, the original diagram was non-wrapping. If $C>1$, one has two possibilities to add two distinct spectator lines without introducing new handles or crossings, that separate the two connected pieces. One of them obstructs the wrapping path. It is possible to add these spectator lines such that they connect the operators with each other. This is possible since we demand that each connected piece was connected to both operators. Restoring the wrapping lines would force 
one to remove one of the spectator lines, since otherwise it had to be crossed, but the other spectator line can be kept, and hence the diagram would be non-wrapping for $C>1$.

In Appendix $\mathrm{A}$ we show that for a theory with vertices with $k+2$ legs that are of order $g^{k}$ in the YM coupling constant, planar wrapping interactions of order $K$ in the YM coupling constant exist for $K \geq 2 R$. As long as $K \leq$ $2(2 R-1)$ they can be constructed from non-wrapping diagrams by adding a single line that runs along the wrapping path. This observation might be useful in explicit computer based calculations like [35, putting the analysis at higher order $(K \leq 2(2 R-1))$ on an equal footing with the critical case $(K=2 R)$.

\subsection{The encoding of the topology of planar wrapping diagrams by the spectator fields}

Restricting to genus $h \leq 1$ contributions to the Green function $V_{2 R}$, one finds from the considerations in Subsection 3.2 that a necessary and sufficient condition for a diagram to be wrapping [non-wrapping] is that there exists no

[at least one] possibility to add a pair of spectator fields $\psi \hat{\psi}$ to the diagram such that the crossings of their connection line with the other field lines do not require adding one handle. We should remark that in this statement the restriction to $h \leq 1$ is essential. For $h \geq 2$ one can find counter-examples to this statement, in which the handles allow one to add spectator lines to wrapping diagrams without changing their genera. We have depicted some examples in Fig. 7 .

Instead of adding one pair of spectator fields, one can directly put $R$ pairs of spectator fields into the $R$ positions between the $\phi_{n_{r}}$ of each of the two operators $\varnothing e 2 R$. No crossings between spectator connections are allowed. Keeping fixed the position of the $\psi$, there are $R$ possible cyclic permutations for the $\hat{\psi}$. This means that to each of the $R^{2}$ contributions to $\left(\varnothing 1 R, V_{2 R}, \varnothing 2 R\right)$ that come from the cyclic permutations of the operators $\varnothing e 2 R$ in (2.1), one has to associate $R$ variants by cyclic permutations only of the spectators within $\varnothing 22 R$, keeping fixed the fields $\phi_{n_{r}}$ in its trace.

With the help of these $R$ variants of the spectator structure, one can now distinguish between wrapping and non-wrapping diagrams. In the case of a wrapping diagram, all pairwise connections between the spectators in each of the $R$ variants crosses at least one other field line. In case of a non-wrapping diagram, there is at least one variant in which one spectator line obstructs the 
unoccupied wrapping path such that only up to $R-1$ spectator lines cross other lines.

In the case of planar contributions $\left(\varnothing 1 R, V_{2 R}, \varnothing 2 R\right)^{(0)}$ to the 2-point function, each connection of a pair of spectators that crosses other lines increases the genus $H$ by one. It is important to stress that only in the planar case one necessarily has to add a handle for each spectator line that crosses other lines. For higher genus diagrams this correspondence breaks down as is shown by some examples in Fig. 7. One reason for this is that one handle can be used not only to cross another line, but even to resolve an additional crossing between lines that run along this handle. For $H \geq 1$ there seems to be no universal way to identify the connections that are responsible for the genus reduction.

The result from the above considerations is that a diagram $D_{\mathrm{w}, 2 R}^{K(1)}$ is a planar wrapping diagram if the genus of the corresponding diagram of the 2-point function $\left(\varnothing 12 R, D_{\mathrm{w}, 2 R}^{K(1)}, \varnothing 22 R\right)$ with $R$ spectator pairs is always increased by $R$, i.e. to $H=R$. Here we have introduced the modified 2-point function $\left(\varnothing 12 R, V_{2 R}, \varnothing 22 R\right)$, where the difference in the number of elementary fields at both $\emptyset e 2 R$ and $V_{2 R}$ is given by the number of spectator fields. In case of genus $H \geq 1$, this statement is sufficient for a diagram to be wrapping, but it is not necessary, as argued above.

The classification via spectators is now used to project out the wrapping contributions. For this purpose, consider the connected $(C=1)$ genus $h=1$ contributions $V_{2 R}^{(1)}$ to $V_{2 R}$. They generate planar wrapping contributions and genus $H=1$ non-wrapping contributions to the 2-point function $\left(\varnothing 1 R, V_{2 R}, \varnothing 2 R\right)$. We now add $R$ pairs of spectators in $R$ different ways as described above and obtain the modified 2-point function $\left(\varnothing 12 R, V_{2 R}, \varnothing 22 R\right)$. We focus on one of its contributions generated by $V_{2 R}^{(1)}$. If all $R$ cyclic permutations of the $R$ spectator fields added to one of the operators, lets say $\hat{\psi}$ in $\varnothing 22 R$, lead to genus $H=R$ contributions, the contribution is a wrapping one. Applying this procedure in case of non-wrapping diagrams, one finds contributions of genus $H=R-1, \ldots R+1$.

$H=R-1$ : One spectator line obstructs the unoccupied wrapping path. Another spectator line does not cross the other field lines because of the single handle in the $h=1$ contributions.

$\underline{H=R}$ : Either one spectator line obstructs the unoccupied wrapping path and all other spectator lines cross other field lines, or the wrapping path remains unobstructed and one spectator line does not cross the other field lines because 


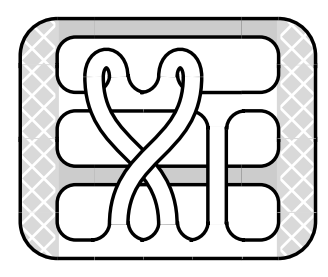

(a)

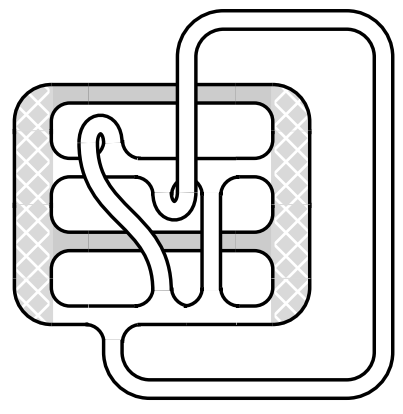

(b)

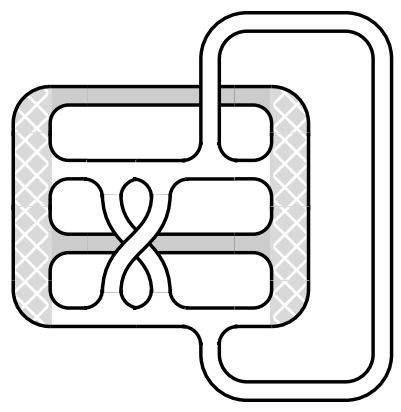

(c)

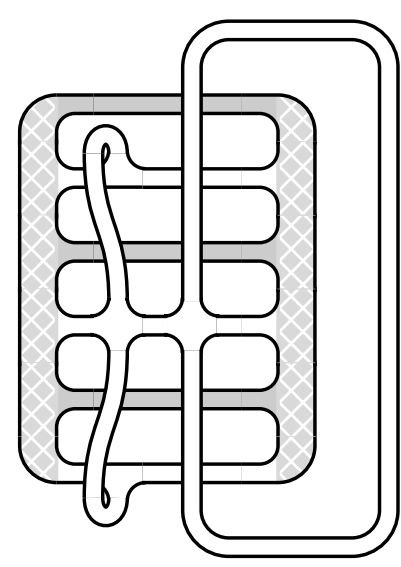

(d)

Figure 7: Some examples of higher genus diagrams with $R=2$ (a) (c) and $R=3(\mathrm{~d})$. Their spectator connections are depicted in gray. A genus $H=1$ non-wrapping diagram (a). A genus $H=1$ wrapping diagram (b) where both spectator connections increase the genus $H$ by one to $H=3$. A genus $H=1$ wrapping diagram (c) where only the upper spectator connection increases the genus $H$ by one to $H=2$. No further handle has to be added for the downer spectator line to resolve its crossings, since this is done by the handle that resolves the crossing between the two field lines. A genus $H=2$ wrapping diagram (d), in which a non-planar self energy contribution of an internal line is responsible for the genus reduction. The two handles for the other field lines resolve the crossings of the line that runs along the wrapping path. 
of the single handle.

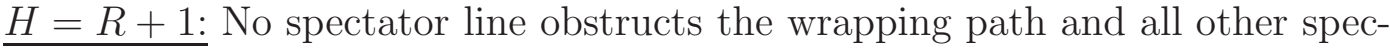
tator lines cross other field lines.

One thus has the relation

$\left(\varnothing 12 R, V_{2 R}^{(1)}, \varnothing 22 R\right)=\sum_{\delta=-1}^{1}\left(\varnothing 12 R, V_{\mathrm{nw}, 2 R}^{(1)}, \varnothing 22 R\right)^{(R+\delta)}+\left(\varnothing 12 R, V_{\mathrm{w}, 2 R}^{(1)}, \varnothing 22 R\right)^{(R)}$.

for the $C=1$ contributions to $V_{2 R} \cdot{ }^{4}$ The above equation is now evaluated as follows: one first adds the $R$ spectator pairs to the operators $\varnothing e 2 R$, only ensuring that their connections never cross each other. Then, for a given contribution to $V_{2 R}$ one performs all contractions of the $\phi_{n_{r}}$, using the rules (B.5).

In this way, one obtains an expression in which the $R$ spectator pairs are distributed among a number of $1 \leq T \leq 2 R$ traces. These distributions encode a classification of the diagrams. To see this, we interpret each trace $\left(\psi_{1} \ldots \psi_{s_{t}}\right)$ over $s_{t}$ spectator fields as a vertex with $s_{t}$ directed legs. The direction distinguishes legs associated to $\psi$ from legs which represent $\hat{\psi}$. Each pairwise contraction is regarded as a propagator connecting an outgoing with an ingoing leg of these vertices. As usual, these diagrams can be classified by the number of vertices, in this case given by $T$, the number of connected pieces $C$, the number of loops $L$. The number of propagators is fixed to $P=R$ and the number of external legs is $E=0$. In this picture, the removal of a spectator pair corresponds to a removal of a propagator. The execution of a contraction between pairs either becomes the fusion of two vertices or the fission of a single vertex into two, depending on whether the propagator contracts two legs at distinct vertices or at only one vertex. The diagrams that are generated from wrapping contributions are now identified as follows: Each removal of a pair $\psi, \hat{\psi}$ must decrease the genus $H$ by one and hence lead to an additional factor $N^{2}$. Thus, each removal of a spectator pair must increase the trace number $T$ by one, compared to the case where this pair is kept and finally contracted. Each trace at the very end contributes a factor $(\mathbb{1})=N$. Another $N$-dependent factor comes from changing the normalization of the operators (2.1) that is required when the number of fields is changed. Furthermore, the above given issues of the spectator diagrams should remain true if all cyclic permutations of the $\hat{\psi}$ are taken into account. Hence, for the spectator diagrams of planar wrapping contributions a necessary condition is that no contractions must occur within a

\footnotetext{
${ }^{4}$ For $C \geq 2$, the sum includes $\delta \leq-2$ and the wrapping contribution is absent.
} 
single trace. Otherwise, the removal of this pair of spectators would not change the genus. Instead of avoiding the fusion of two traces the fission of that trace would be inhibited. Since such a configuration must not occur in any of the cyclic permutations, the spectators within one trace must only be of one type, either $\psi$ or $\hat{\psi}$. Furthermore, the increasing of the trace number by removing one spectator pair must be independent of the contraction or removal of other spectators. This forbids that the connections form any closed loops since, after contracting some of them, one always would end up in a contraction of one spectator pair within a single trace. The spectator diagrams of wrapping contributions are therefore always tree-level and consist of $1 \leq C \leq R$ separate pieces. Any contraction of a pair $\psi, \hat{\psi}$ is an application of the fusion rule (B.5) such that, after having contracted all spectator pairs, $C$ separate traces (1) remain, leading to a factor $N^{C}$. On the other hand, removing all pairs $\psi, \hat{\psi}$, one is left with $T$ traces $(\mathbb{1})$, contributing $N^{T}$. A factor $N^{R}$ has to be considered for the change in the normalization of the operators. According to the last equation in (A.2), in the special case of tree-level diagrams one has the relation $R=T-C$, and hence concludes:

Each tree-level spectator diagram that is built out of $C$ connected pieces contributes a factor $N^{C}$ if the spectators are kept and it contributes a factor $N^{2 R+C}$ if the spectators are removed.

In our case $C$ has to be maximal, i.e. $C=R$. One can find several arguments for this statement. First of all, removing all the spectator pairs, one obtains a planar diagram, and planar diagrams contribute to the leading power in the $\frac{1}{N}$ expansion. This means, if $C$ were not maximal for the planar wrapping diagrams, their contribution would be subdominant. Secondly, one can regard a diagram $D_{2 R}^{K(h)}$ as an effective vertex which is proportional to $\sqrt{\lambda}^{K}$. The dependence on $N$ one should associate with such a vertex can be interpreted as the $N$ power which has to be absorbed into an effective 't Hooft coupling that is later set to 1 . For a planar vertex with $2 R$ external legs one has to absorb a factor $\sqrt{N}^{2 R-2}$, i.e. the $N$ dependence is given by its reciprocal value $N^{1-R}$. When this effective vertex contains $h$ handles, and it is contracted with a planar effective vertex in a planar way, the number of traces is reduced by $h$. This means, compared to the case of a planar diagram which contains two planar vertices, the power in $N$ found from the traces is reduced by $h$. Therefore, to remove a factor $N^{2}$ for each handle in the final result, one has to change the $N$ dependent normalization of an effective vertex of genus $h$ to $N^{1-R-h}$. That means, in case of planar wrapping contributions which have 
$h=1$, including the traces over all spectator pairs, and the normalization of the two operators $\emptyset e 2 R$ taken from (2.1), the total $N$ dependence is $N^{2+C-3 R}$. This factor should be equal to $N^{2-2 H}$ with $H=R$ (see (3.3) ), such that one deduces $C=R .^{5}$

From the above considerations it follows that one can uniquely identify the wrapping diagrams that contribute to the planar 2-point function by finding the spectator structure

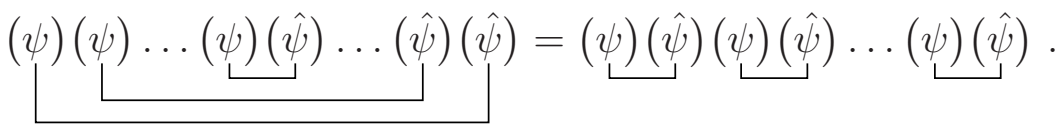

The issue that all traces contain only one spectator field enables one in principle

to directly project out the planar wrapping contributions generated by $V_{2 R}^{(1)}$. One has to find an appropriate matrix for $\psi$ and $\hat{\psi}$ such that traces which include more than one spectator field vanish. Such traces are always included in the remaining genus $h=1$ non-wrapping contributions. This issue is discussed in Section 6.

\section{Planar contributions to the 2-point function}

\subsection{Non-interacting case}

The simplest contribution to the 2-point function $\left(\varnothing 1 R, V_{2 R}, \varnothing 2 R\right)$ is given by a pairwise planar $(h=0)$ connection of all the legs of the two operators without any interactions. The corresponding contribution to $V_{2 R}$ is given by the diagram $D_{2 R}^{0(0)}$ which is a direct product of free propagators that connect the fields with label $r$ and $2 R-r+1, r=1, \ldots R$. The color part of this contribution, in case of a $U(N)$ gauge group, is given by

$$
\left(\varnothing 1 R, D_{2 R}^{0(0)}, \varnothing 2 R\right) \rightarrow R^{2} \frac{1}{N^{R-2}}\left(a_{1} \ldots a_{R}\right)\left(a_{R+1} \ldots a_{2 R}\right)=R^{2} N(\mathbb{1})=R^{2} N^{2}
$$

\footnotetext{
${ }^{5}$ This argument is only true if the contractions of the $\phi_{n_{r}}$ never generate powers in $N$. According to $[\mathbb{B} .3)$, this would happen if two contractible fields $\phi_{n_{r}}$ become direct neighbours within one trace without further fields. That this cannot happen here is guaranteed by the spectators that separate all $\phi_{n_{r}}$ from each other.
} 


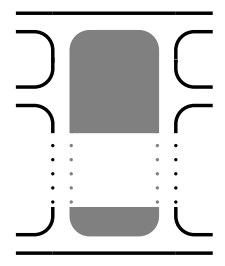

Figure 8: A generic planar connected contribution to $V_{2 R}$. The picture shows that a planar contribution contracts the two index lines of one external line with one of the index lines of the two neighboured external lines. This corresponds to a contraction of the indices of the representation matrices. The gray-filled structure in the middle represents any planar diagram. It has no influence on the index lines that belong to the external legs. Thus all planar contributions consists of a single trace over the representation matrices.

where the factor $R^{2}$ stems from the $R^{2}$ pairs of cyclic permutations of the fields in each of the two operators $\varnothing 1 R$. The second equality in (4.1) follows after applying the fusion rules for the traces $(\underline{B .5})$ and the identity (B.3) with $a_{0}=0$.

The $N$ dependent prefactor is the square of the normalization of the operators in (2.1), and it is chosen such that the above result is of the order $N^{2}$. A genus $H$ contribution to $\left(\varnothing 1 R, V_{2 R}, \varnothing 2 R\right)$ will then be of the order $N^{2-2 H}$.

\subsection{Generic planar connected contributions to $V_{2 R}$}

Consider the planar connected contributions to $V_{2 R}$ denoted as $V_{2 R}^{(0)}$. Their general form is shown in Fig. 8 .

From the fact that in the planar case lines must not cross in the corresponding ribbon graphs, it is obvious that they can always be represented as an effective vertex of the form

$$
V_{2 R}^{(0)}=f_{2 R}^{(0)}(\sqrt{\lambda}) \frac{1}{N^{R-1}}\left(a_{2 R} \ldots a_{1}\right),
$$

where $f_{2 R}^{(0)}(\sqrt{\lambda})$ captures the individual properties of the diagrams, i.e. their coupling dependence and the contributions from the loop integrals. The $N$ dependence has been fixed such that any planar diagram including this vertex is of the order $N^{2}$. 
Including $R$ spectator pairs $\psi \hat{\psi}$, the generic planar contribution (4.2) to the 2-point function is given by

$$
\begin{aligned}
& \left(\varnothing 12 R, V_{2 R}^{(0)}, \varnothing 22 R\right) \\
& \propto\left(a_{1} \psi a_{2} \ldots \psi a_{R} \psi\right)\left(a_{R} \ldots a_{1} a_{2 R} \ldots a_{R+1}\right)\left(\hat{\psi} a_{R+1} \hat{\psi} \ldots a_{2 R-1} \hat{\psi} a_{2 R}\right) \\
& + \text { cycl. perm. } \hat{\psi} \\
& =(\psi) \ldots(\underset{L}{\psi}(\underset{\psi}{\psi})(\hat{\psi}) \ldots(\hat{\psi})+\text { cycl. perm. } \hat{\psi}
\end{aligned}
$$

where in the first equality we have used the invariance of the trace under cyclic permutations for a reordering of (4.2). The contribution given explicitly in the second equality of (4.3) is the one that identifies the diagram as non-wrapping, since the removal of the spectator pair in the single trace does not increase the power in $N$ and hence does not decrease the genus of the diagram. The connection line of this spectator pair obstructs the wrapping path. The other contributions to (4.3) are of the form

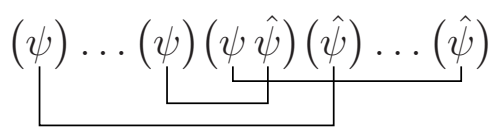

In contrast to first contribution in the second line of (4.3), the removal of any spectator pair increases the power in $N$ by two and hence reduces the genus of the corresponding diagram by one. In these contributions, no spectator line obstructs the wrapping path.

\subsection{Generic planar wrapping contributions to $V_{2 R}$}

Consider the planar wrapping contributions to $V_{2 R}$ denoted by $V_{\mathrm{w}, 2 R}^{(1)}$. In a genus expansion of the 2-point function $\left(\varnothing 1 R^{\prime}, V_{2 R}, \varnothing 2 R^{\prime}\right)$ they contribute at planar level if $R^{\prime}=R$, but all their contributions become genus $H=1$ for $R^{\prime}>R$. The most general planar wrapping contribution is obtained by taking the most general planar diagram and then filling the wrapping path with the most general planar structure. One then arrives at the ribbon graph shown in Fig. 9] 


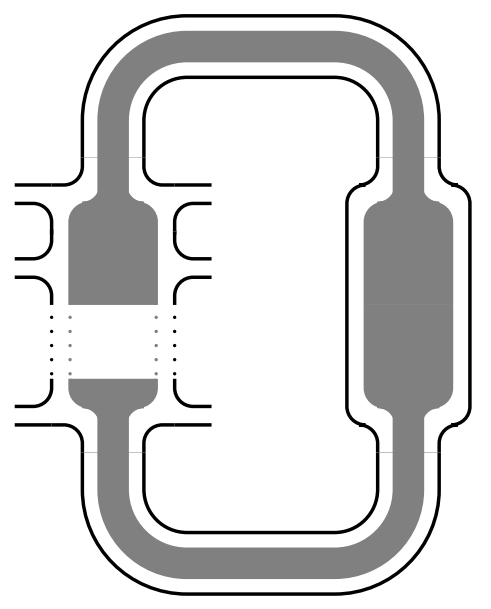

Figure 9: A generic planar wrapping contribution to $V_{2 R}$. The occupation of the wrapping path separates the ingoing and outgoing fields from each other and breaks the single trace of ordinary planar diagrams in Fig. 8 into two traces. The gray-filled structure in the middle represents any planar diagram. It has no influence on the lines that belong to the external legs. Thus all planar wrapping contributions consists of a product of two separate traces over the representation matrices for the ingoing and outgoing legs. 
From the restriction that lines must not cross in this graph, one can read off the general effective vertex for a generic planar wrapping diagram. It is given by

$$
V_{\mathrm{w}, 2 R}^{(1)}=f_{\mathrm{w}, 2 R}^{(1)}(\sqrt{\lambda}) \frac{1}{N^{R}}\left(a_{R} \ldots a_{1}\right)\left(a_{2 R} \ldots a_{R+1}\right),
$$

where $f_{\mathrm{w}, 2 R}^{(1)}(\sqrt{\lambda})$ captures the individual properties of the wrapping diagrams, i.e. their coupling dependence and the contributions from the loop integrals. The $N$ dependence has been fixed such that any diagram with two operators $\varnothing e 2 R^{\prime}, R^{\prime}>R$ including this vertex is of the order $N^{0}$. The vertex (4.5) is obviously invariant under separate cyclic permutations of the ingoing and outgoing legs. This is even obvious in Fig. 9, since these cyclic permutations do not change the structure represented by the gray-filled region. The symmetry found for wrapping diagrams is consistent with the observation, that the wrapping handle is sufficient to resolve all those crossings within the ingoing and outgoing external legs of a given diagram $D_{2 R}^{K(1)}$ that can be traced back to cyclic permutations. This can be easily understood, looking at Fig. 3. and adding the wrapping handle that connects the regions above and below the vertex at $\infty$.

Including $R$ spectator pairs $\psi \hat{\psi}$, the 2-point function $\left(\varnothing 12 R, V_{\mathrm{w}, 2 R}^{(1)}, \varnothing 22 R\right)$ of the generic planar wrapping contribution (4.5) is given by

$$
\begin{aligned}
& \left(\varnothing 12 R, V_{\mathrm{w}, 2 R}^{(1)}, \varnothing 22 R\right) \\
& \propto R\left(a_{1} \psi a_{2} \ldots \psi a_{R} \psi\right)\left(a_{R} \ldots a_{1}\right)\left(a_{2 R} \ldots a_{R+1}\right)\left(\hat{\psi} a_{R+1} \hat{\psi} \ldots a_{2 R-1} \hat{\psi} a_{2 R}\right) \\
& \quad=R(\psi) \ldots(\hat{\psi})(\hat{\psi})(\hat{\psi})(\hat{\psi}) \ldots(\hat{\psi})
\end{aligned}
$$

where the factor $R$ in the first equality is generated by the cyclic permutations of the $\hat{\psi}$ that in this case do not lead to different contributions. The final result of (3.4) is the one that identifies the diagram as planar wrapping, since each removal of a spectator pair increases the number of traces and hence the power in $N$ by one. With the additional factor of $N$ from the change in the normalization this leads to a factor $N^{2}$, showing the decreasing of the genus $H$ of the diagram by one. 


\subsection{Generic planar wrapping contributions to $V_{2 R}$ in- cluding flavor}

We extend the effective vertex (4.5) of planar wrapping interactions by considering theories which include flavor degrees of freedom such that the flavor structure is described by Kronecker $\delta$ s. In the subsector of composite operators which contain no trace terms in their flavor indices ${ }^{6}$ one can write down a rather simple expression for this vertex. It is given by

$$
\begin{aligned}
V_{\mathrm{w}, 2 R}^{(1)}= & \frac{1}{N^{R}}\left(a_{R} \ldots a_{1}\right)\left(a_{2 R} \ldots a_{R+1}\right) \\
& \times \sum_{\pi \in \frac{S_{R}}{C_{R}}} \frac{f_{\pi, 2 R}^{(1)}(\sqrt{\lambda})}{R} \sum_{\omega \in C_{R}} \delta_{m_{\omega(\pi(2 R))}}^{m_{1}} \delta_{m_{\omega(\pi(2 R-1))}}^{m_{2}} \ldots \delta_{m_{\omega(\pi(R+1))}}^{m_{R}},
\end{aligned}
$$

where $f_{\pi, 2 R}^{(1)}(\sqrt{\lambda})$ captures the individual properties of the diagrams, and $m_{r}$ is the corresponding flavor index carried by the leg with color index $a_{r}$. Some remarks should be made. First of all, the above expression is valid to arbitrary high order in $\lambda$. Secondly, the vertex is chosen such that its flavor dependence is invariant under separate cyclic permutations applied to the two pairs of $R$ ingoing and outgoing legs. Since the color dependence respects the same symmetry, it is a symmetry of the complete vertex.

The choice of this effective vertex enables one to reduce the number of coefficient functions from $R$ ! in the original vertex to $(R-1)$ !. This reduction should be useful in practical calculations. Instead of explicitly computing the coefficient functions by summing up the diagrams of the perturbation expansion, one could try to determine the coefficient functions by fitting them to available data. In this case, the chosen effective vertex should of course contain the minimum number of coefficient functions. The choice of (4.7) would be a first step. As a second step, one should try to use additional symmetries, unitarity, non-renormalization theorems, etc. to further reduce the number of independent coefficient functions.

\footnotetext{
${ }^{6}$ This is true for instance in the $S U(2)$ subsector of $\mathcal{N}=4 \mathrm{SYM}$, consisting of two scalars $X=\frac{1}{\sqrt{2}}\left(\phi_{1}+i \phi_{2}\right), Y=\frac{1}{\sqrt{2}}\left(\phi_{3}+i \phi_{4}\right)$.
} 


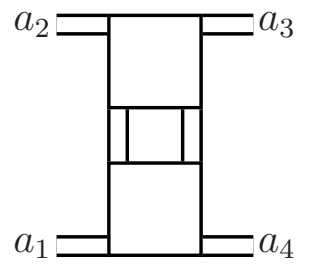

(a)

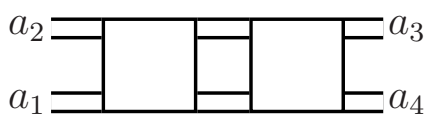

(b)

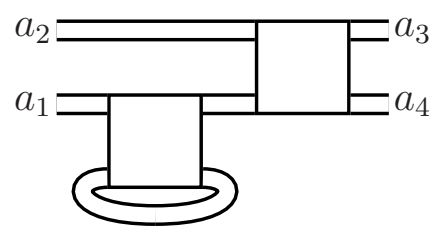

(c)

Figure 10: The three possible types of connected $(C=1)$ Feynman diagrams occurring at the perturbative order $g^{4}$ in $V_{4}$.

\section{The toy model}

Let us consider the following toy-example of a single scalar field in the adjoint representation of $U(N)$, with a standard kinetic term and an interaction Lagrangian:

$$
S_{\mathrm{int}}[\phi]=\frac{g^{2}}{4 !} \operatorname{tr}(\phi \phi \phi \phi) .
$$

From here one can derive the interaction vertex as

$$
\begin{aligned}
V^{a_{1} a_{2} a_{3} a_{4}}=\frac{g^{2}}{3 !}[ & \left(a_{1} a_{2} a_{3} a_{4}\right)+\left(a_{1} a_{2} a_{4} a_{3}\right)+\left(a_{1} a_{3} a_{2} a_{4}\right) \\
& \left.+\left(a_{2} a_{1} a_{3} a_{4}\right)+\left(a_{1} a_{4} a_{2} a_{3}\right)+\left(a_{1} a_{4} a_{3} a_{2}\right)\right]
\end{aligned}
$$

where we have used the abbreviation (2.2) for the traces. Due to the cyclic invariance of the trace, only six inequivalent permutations out of $4 !=24$ possible permutations remain.

We compute the $\propto g^{4}$ contribution to the 2-point function of the composite operator $\varnothing 2=\operatorname{tr}\left(\phi^{2}\right)$ in perturbation theory. As derived in Appendix A this order $(K=4)$ is precisely the critical case for this operator $(R=2)$, where wrapping issues appear first. This example reproduces, in a fairly simplified version, one of the situations one encounters in the more complicated case of $\mathcal{N}=4$ SYM.

In a theory with vertices only of type (5.1) at order $g^{4}$, there are only three possible types of connected $(C=1)$ Feynman diagrams contributing to $V_{4}$. We 
have depicted them in Fig. 10] Combining the six terms of the two 4-vertices (5.2) together, and considering all possible permutations of the external legs, one gets $(4 !)^{2}$ diagrams for the $t$ - and the $u$-channel (Fig. 10(a) and for the $s$-channel (Fig. 10(b). The self energy contribution Fig. 10(c) leads to $4(4 !)^{2}$ diagrams. All terms can be grouped together into 17 different color structures that enter the full vertex obeying crossing symmetry. The result reads

$$
\begin{aligned}
& V_{4, \text { sym. }}^{a_{1} a_{2} a_{3} a_{4}}=\frac{2 g^{4}}{(3 !)^{2}}\left[N \left\{\left(\alpha_{t}+\alpha_{s}+8 \gamma^{\prime}\right)\left[\left(a_{4} a_{1} a_{2} a_{3}\right)+\left(a_{4} a_{3} a_{2} a_{1}\right)\right]\right.\right. \\
& +\left(\alpha_{t}+\alpha_{u}+8 \gamma^{\prime}\right)\left[\left(a_{4} a_{1} a_{3} a_{2}\right)+\left(a_{4} a_{2} a_{3} a_{1}\right)\right] \\
& \left.+\left(\alpha_{u}+\alpha_{s}+8 \gamma^{\prime}\right)\left[\left(a_{4} a_{3} a_{1} a_{2}\right)+\left(a_{4} a_{2} a_{1} a_{3}\right)\right]\right\} \\
& +\left(\alpha_{t}+\alpha_{u}+\alpha_{s}+3 \gamma^{\prime}\right)\left\{\left(a_{1} a_{2} a_{3}\right)\left(a_{4}\right)+\left(a_{1} a_{3} a_{2}\right)\left(a_{4}\right)\right. \\
& +\left(a_{1} a_{2} a_{4}\right)\left(a_{3}\right)+\left(a_{2} a_{1} a_{4}\right)\left(a_{3}\right) \\
& +\left(a_{1} a_{3} a_{4}\right)\left(a_{2}\right)+\left(a_{3} a_{1} a_{4}\right)\left(a_{2}\right) \\
& \left.+\left(a_{2} a_{3} a_{4}\right)\left(a_{1}\right)+\left(a_{3} a_{2} a_{4}\right)\left(a_{1}\right)\right\} \\
& +\left(4 \alpha_{t}+\alpha_{u}+\alpha_{s}\right)\left(a_{1} a_{4}\right)\left(a_{2} a_{3}\right) \\
& +\left(\alpha_{t}+4 \alpha_{u}+\alpha_{s}\right)\left(a_{1} a_{3}\right)\left(a_{2} a_{4}\right) \\
& \left.+\left(\alpha_{t}+\alpha_{u}+4 \alpha_{s}\right)\left(a_{1} a_{2}\right)\left(a_{3} a_{4}\right)\right] \text {, }
\end{aligned}
$$

where $\alpha_{t}, \alpha_{u}, \alpha_{s}, \gamma^{\prime}$ describe the spacetime factors, including integrals over the positions of the two vertices and depending on the coordinates $x_{1}, x_{2}$ and $y_{1}$, $y_{2}$ of the ingoing and outgoing fields, respectively. To obtain (5.3) we have repeatedly used the $U(N)$ fusion and fission rules (B.5) and the trace of the identity $(\mathbb{1})=N$.

To match our definition of the Green function, when used as a building block of the 2-point function, which is given in Subsection 2.3 (see also Fig. 5), we divide out the cyclic permutations acting separately on the incoming and outgoing legs. In this case these are simply the exchanges $a_{1} \leftrightarrow a_{2}$ and $a_{3} \leftrightarrow a_{4}$, under which the 17 color terms in (5.3) boil down to 6 distinct structures. We obtain the building block

$$
\begin{aligned}
V_{4}^{a_{1} a_{2} a_{3} a_{4}}=\frac{2 g^{4}}{(3 !)^{2}}[ & N\left\{(\alpha+\beta+8 \gamma)\left(a_{4} a_{3} a_{2} a_{1}\right)+(\alpha+4 \gamma)\left(a_{4} a_{1} a_{3} a_{2}\right)\right\} \\
+ & (2 \alpha+\beta+3 \gamma)\left\{\left(a_{1} a_{2} a_{3}\right)\left(a_{4}\right)+\left(a_{1} a_{3} a_{4}\right)\left(a_{2}\right)\right\} \\
+ & \left.\frac{1}{2}\left\{(5 \alpha+\beta)\left(a_{1} a_{4}\right)\left(a_{2} a_{3}\right)+(\alpha+2 \beta)\left(a_{1} a_{2}\right)\left(a_{3} a_{4}\right)\right\}\right] .
\end{aligned}
$$

Furthermore, we have identified $x_{1}=x_{2}=x$ and $y_{1}=y_{2}=y$ such that 
$\alpha_{t}, \alpha_{u} \rightarrow \alpha, \alpha_{s} \rightarrow \beta$ and $\gamma^{\prime} \rightarrow \gamma$. The coefficients $\alpha, \beta, \gamma$ contain the corresponding 3-loop integrals. With the coordinate expression for the propagator

$$
I_{x y}=\frac{\Gamma\left(\frac{d}{2}-1\right)}{4 \pi^{\frac{d}{2}}} \frac{1}{\left((x-y)^{2}-i \epsilon\right)^{\frac{d}{2}-1}}
$$

they read in $d=4-2 \varepsilon$ dimensions

$$
\alpha=\int \mathrm{d}^{d} u \mathrm{~d}^{d} v I_{x u} I_{x v} I_{u v}^{2} I_{u y} I_{v y}, \quad \beta=\int \mathrm{d}^{d} u \mathrm{~d}^{d} v I_{x u}^{2} I_{u v}^{2} I_{v y}^{2}, \quad \gamma=0 .
$$

In the last equality the vanishing of all tadpole-type graphs in dimensional regularization has been used. Moreover, they would not in any case affect the analysis of planar wrapping diagrams, since it is straightforwardly seen that they never lead to planar wrapping contributions. Explicit results for $\alpha$ and $\beta$ can be obtained by the Gegenbauer $x$-space technique (see (2.20) in [37]), and for $\beta$ more easily by the methods based on uniqueness 38 .

The 2-point function of the operator $\varnothing 2=\operatorname{tr}\left(\phi^{2}\right)$ is obtained from (5.4) by contracting the indices $a_{1}, a_{2}$ and $a_{3}, a_{4}$ with tensors proportional to $\delta_{a_{2}}^{a_{1}}$ and $\delta_{a_{4}}^{a_{3}}$, respectively. This is obvious after rewriting $\operatorname{tr}\left(\phi^{2}\right)$ as $\phi_{a} \phi_{b} \operatorname{tr}\left(T^{a} T^{b}\right)$ and using (B.1). The color part reduces to either $N^{4}$ or $N^{2}$ if the corresponding diagram is of genus $H=0$ or $H=1$, respectively. This is in accord with the genus expansion of the 2-point function in Subsection 2.3.

The planar contributions to the 2-point function are identified as the ones that contain the color structures $\left(a_{4} a_{3} a_{2} a_{1}\right)$ and $\left(a_{1} a_{2}\right)\left(a_{3} a_{4}\right)$. By drawing the diagrams, one can check that the structure with a single trace is associated to the non-wrapping diagrams, while the double trace contribution takes care of the planar wrapping diagrams. This coincides with the generic results (4.2) and (4.5).

The identification of planar wrapping diagrams is simple in this special case, where the number of diagrams is small enough to draw all of them. We can therefore explicitly check our procedure based on spectator insertions as introduced in Subsection 3.2, adding two pairs of spectator fields such that the modified 2-point function becomes

$$
\left(a_{1} \psi a_{2} \psi\right) V_{4}^{a_{1} a_{2} a_{3} a_{4}}\left(\hat{\psi} a_{3} \hat{\psi} a_{4}\right)+\text { cycl. perm. Øe } 2+\text { cycl. perm. } \hat{\psi} \text {. }
$$

Here, cyclic permutations within $\emptyset e 2$ corresponds to the 4 possible combinations obtained by the changes $a_{1} \leftrightarrow a_{2}, a_{3} \leftrightarrow a_{4}$ within the two operators $\varnothing e 2$. 
The terms in (5.4) lead to the distinct spectator structures

$$
\begin{aligned}
& \left(a_{4} a_{3} a_{2} a_{1}\right) \rightarrow(\underbrace{\psi})(\underset{\psi}{\psi} \hat{\psi})(\hat{\psi})+(\underbrace{\psi})(\underbrace{\psi} \hat{\psi})(\hat{\psi}),
\end{aligned}
$$

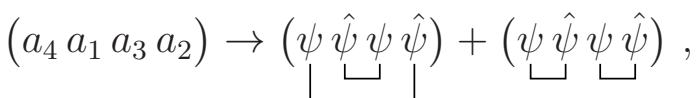

$$
\begin{aligned}
& \left(a_{1} a_{2} a_{3}\right)\left(a_{4}\right) \rightarrow(\underbrace{\psi}_{\llcorner})(\underset{\psi}{\psi} \hat{\psi})+(\underbrace{\psi})(\underbrace{\psi}_{\mathcal{\psi}} \hat{\psi})
\end{aligned}
$$

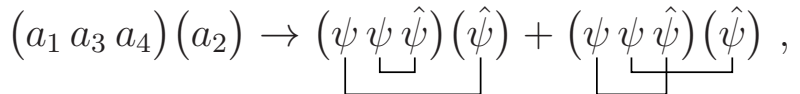

$$
\begin{aligned}
& \left(a_{1} a_{4}\right)\left(a_{2} a_{3}\right) \rightarrow(\underset{\psi}{\psi} \hat{\psi})(\psi \hat{\psi})+(\underline{\psi} \hat{\psi})(\underline{\psi} \hat{\psi}) \\
& \left(a_{1} a_{2}\right)\left(a_{3} a_{4}\right) \rightarrow 2(\psi)(\hat{\psi})(\psi)(\hat{\psi}) .
\end{aligned}
$$

As can be easily seen, the only case in which the traces completely factorize into four single pieces is the wrapping case, in accord with our general arguments in Subsection 3.3

The toy example we presented up to here is interesting, but of course it does not include the full $\mathcal{N}=4$ complexity. In order to get closer to that case,

in a first step we add flavor degrees of freedom to the interaction Lagrangian (5.1) in a simple way, such that the number of terms in the symmetrized interaction vertex (5.2) is not enlarged. In a second step, we then consider the 4 -scalar commutator interaction of the full $\mathcal{N}=4$ theory, which symmetrized interaction vertex contains several terms with different flavor dependence.

\subsection{4-vertex with cyclic symmetric flavor flux}

In our first refinement we take as interaction term

$$
S_{\mathrm{int}}[\phi]=\frac{g^{2}}{4 !} \operatorname{tr}\left(\phi_{m} \phi_{n} \phi_{m} \phi_{n}\right)
$$

where we have introduced flavor indices $m, n=1, \ldots, N_{\mathrm{f}}$. The two flavor lines that enter and leave the vertex at its four legs cross each other as depicted in Fig. 11(a)

Since the structure with crossing flavor lines is invariant under cyclic permutations, the symmetrized vertex consists of six inequivalent terms as in (5.2), 


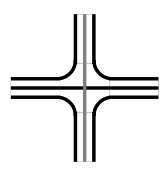

(a)

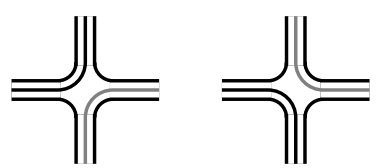

(b)

Figure 11: The flow of a flavor line through a vertex of the types $\operatorname{tr}\left(\phi_{m} \phi_{n} \phi_{m} \phi_{n}\right)$ (a) and $\operatorname{tr}\left(\phi_{m} \phi_{m} \phi_{n} \phi_{n}\right)$ (b). In the second case, the two permutations have to be taken into account in the symmetrized vertex.

but with an additional simple factor for the flavor dependence. One gets

$$
\begin{aligned}
V_{m_{1} m_{2} m_{3} m_{4}}^{a_{1} a_{2} a_{3} a_{4}}=\frac{g^{2}}{3 !}[ & \left(a_{1} a_{2} a_{3} a_{4}\right) \delta_{m_{3}}^{m_{1}} \delta_{m_{4}}^{m_{2}}+\left(a_{1} a_{2} a_{4} a_{3}\right) \delta_{m_{4}}^{m_{1}} \delta_{m_{3}}^{m_{2}} \\
& +\left(a_{1} a_{3} a_{2} a_{4}\right) \delta_{m_{2}}^{m_{1}} \delta_{m_{4}}^{m_{3}}+\left(a_{2} a_{1} a_{3} a_{4}\right) \delta_{m_{4}}^{m_{1}} \delta_{m_{3}}^{m_{2}} \\
& \left.+\left(a_{1} a_{4} a_{2} a_{3}\right) \delta_{m_{2}}^{m_{1}} \delta_{m_{4}}^{m_{3}}+\left(a_{1} a_{4} a_{3} a_{2}\right) \delta_{m_{3}}^{m_{1}} \delta_{m_{4}}^{m_{2}}\right]
\end{aligned}
$$

where $m_{r}$ is the corresponding flavor index carried by the leg with color index $a_{r}$, arranged as in Fig. 10.

The genus expansion is an expansion in powers of $\frac{1}{N}$, the inverse of the number of colors. Adding flavor degrees of freedom does not influence this expansion, it simply extends the result of a single field by multiplying each term by a flavor dependent factor. We consider the 2-point function of the operator $\varnothing 2=\operatorname{tr}\left(\phi_{m} \phi_{m}\right)$ which now include a summation over all flavors. The planar wrapping diagrams turn out to be given by

$$
\frac{g^{4}}{(3 !)^{2}}\left(a_{1} a_{2}\right)\left(a_{3} a_{4}\right)\left[\left(\alpha N_{\mathrm{f}} \delta_{m_{4}}^{m_{1}} \delta_{m_{3}}^{m_{2}}+2 \beta \delta_{m_{3}}^{m_{1}} \delta_{m_{4}}^{m_{2}}\right] .\right.
$$

This result can be brought into the symmetrized form (4.7), where the single coefficient is proportional to $\alpha N_{\mathrm{f}}+2 \beta$.

In the corresponding ribbon graph Fig. 9] the color structure $\left(a_{1} a_{2}\right)\left(a_{3} a_{4}\right)$ has a simple interpretation as the breaking of the single surrounding index line by the structure along the wrapping path. Adding flavor lines to the corresponding diagrams, one finds an interpretation even for the flavor structure in (5.11). A vertex with the flavor flow as given in Fig. 11(a) yields the diagram depicted in Fig. 12(a) A factor $N_{\mathrm{f}}$ can be traced back to a closed flavor loop 


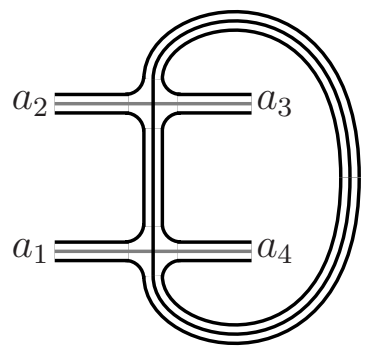

(a)

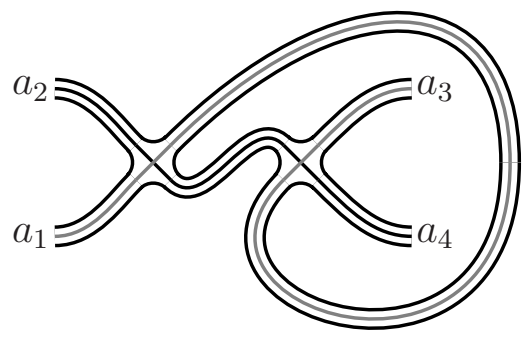

(b)

Figure 12: Contributions to planar wrapping interactions in the presence of flavor. The structure in Fig. 10(a) leads to contributions with a closed flavor loop as shown in (a) The structure in Fig. 10(b) leads to contributions depending on $\delta_{m_{3}}^{m_{1}} \delta_{m_{4}}^{m_{2}}$ as shown in (b).

which is present in diagrams of the type shown in Fig. 10(a). The remaining flavor lines can be associated to the factor $\delta_{m_{4}}^{m_{1}} \delta_{m_{3}}^{m_{2}}$. The other contributions are based on the type of diagrams shown in Fig. 10(b). They do not contain closed flavor loops and can be associated to the flavor structure $\delta_{m_{3}}^{m_{1}} \delta_{m_{4}}^{m_{2}}$, see Fig. 12(b).

\subsection{4-vertex with commutators}

Now we get more into contact with $\mathcal{N}=4 \mathrm{SYM}$ adopting the proper commutator interaction given by

$$
S_{\mathrm{int}}[\phi]=\frac{1}{2} \frac{g^{2}}{4 !} \operatorname{tr}\left[\phi_{m}, \phi_{n}\right]\left[\phi_{m}, \phi_{n}\right]=\frac{g^{2}}{4 !} \operatorname{tr}\left(\phi_{m} \phi_{n} \phi_{m} \phi_{n}\right)-\frac{g^{2}}{4 !} \operatorname{tr}\left(\phi_{m} \phi_{m} \phi_{n} \phi_{n}\right)
$$

where we have introduced the factor $\frac{1}{2}$ for convenience.

The interaction in (5.12) appears as the combination of the previous one (5.9) with another term in which the flavor lines 'repulse' each other, as depicted in Fig. 11(b),

The vertex with repulsing flavor lines is not invariant under all cyclic permutations, but only under those where the legs are shifted by an even number. 
So one has to keep track of two contributions, differing by an odd number of shifts. The explicit form of the vertex turns out to be

$$
\begin{aligned}
V_{m_{1} m_{2} m_{3} m_{4}}^{a_{1} a_{2} a_{3} a_{4}}=\frac{2 g^{2}}{4 !}[ & \left(a_{1} a_{2} a_{3} a_{4}\right)\left(2 \delta_{m_{3}}^{m_{1}} \delta_{m_{4}}^{m_{2}}-\delta_{m_{2}}^{m_{1}} \delta_{m_{4}}^{m_{3}}-\delta_{m_{4}}^{m_{1}} \delta_{m_{3}}^{m_{2}}\right) \\
& +\left(a_{1} a_{2} a_{4} a_{3}\right)\left(2 \delta_{m_{4}}^{m_{1}} \delta_{m_{3}}^{m_{2}}-\delta_{m_{3}}^{m_{1}} \delta_{m_{4}}^{m_{2}}-\delta_{m_{2}}^{m_{1}} \delta_{m_{4}}^{m_{3}}\right) \\
& +\left(a_{1} a_{3} a_{2} a_{4}\right)\left(2 \delta_{m_{2}}^{m_{1}} \delta_{m_{4}}^{m_{3}}-\delta_{m_{3}}^{m_{1}} \delta_{m_{4}}^{m_{2}}-\delta_{m_{4}}^{m_{1}} \delta_{m_{3}}^{m_{2}}\right) \\
& +\left(a_{2} a_{1} a_{3} a_{4}\right)\left(2 \delta_{m_{4}}^{m_{1}} \delta_{m_{3}}^{m_{2}}-\delta_{m_{3}}^{m_{1}} \delta_{m_{4}}^{m_{2}}-\delta_{m_{2}}^{m_{1}} \delta_{m_{3}}^{m_{3}}\right) \\
& +\left(a_{1} a_{4} a_{2} a_{3}\right)\left(2 \delta_{m_{2}}^{m_{1}} \delta_{m_{4}}^{m_{3}}-\delta_{m_{3}}^{m_{1}} \delta_{m_{4}}^{m_{2}}-\delta_{m_{4}}^{m_{1}} \delta_{m_{3}}^{m_{2}}\right) \\
& \left.+\left(a_{1} a_{4} a_{3} a_{2}\right)\left(2 \delta_{m_{3}}^{m_{1}} \delta_{m_{4}}^{m_{2}}-\delta_{m_{2}}^{m_{1}} \delta_{m_{4}}^{m_{3}}-\delta_{m_{4}}^{m_{1}} \delta_{m_{3}}^{m_{2}}\right)\right] .
\end{aligned}
$$

The identification of the planar wrapping diagrams proceeds as in Subsection 5.1. After a tedious but straightforward computation of the flavor factor one ends up with the result

$$
\frac{g^{4}}{2(3 !)^{2}}\left(a_{1} a_{2}\right)\left(a_{3} a_{4}\right)\left[\left(\alpha-2 \beta+\beta N_{\mathrm{f}}\right) \delta_{m_{2}}^{m_{1}} \delta_{m_{4}}^{m_{3}}+(\alpha+\beta) \delta_{m_{3}}^{m_{1}} \delta_{m_{4}}^{m_{2}}+2 \alpha\left(N_{\mathrm{f}}-2\right) \delta_{m_{4}}^{m_{1}} \delta_{m_{3}}^{m_{2}}\right] \text {. }
$$

Its flavor dependence can again be reconstructed by taking into account all the possible flavor flows generated by combining the vertices in Fig. 11] This result can be brought into the symmetrized form (4.7), where the single coefficient is proportional to $2 \alpha N_{\mathrm{f}}-3 \alpha+\beta$.

\section{A candidate for $\psi$}

In this Section we want to show how one can project out the planar wrapping contributions from all contributions to the 2-point function that contain $V_{2 R}^{(1)}$, by choosing suitable matrices for the spectator pairs $\psi, \hat{\psi}$. The planar wrapping

contributions have the property that they are the only contributions to $V_{2 R}^{(1)}$ that generate the spectator structure (3.4) where the trace of all spectator fields is taken separately. If one could find a non traceless matrix with the property that its positive powers $p$ are traceless up to a sufficiently high order $p_{0}, 2 \leq p \leq p_{0}$, then one could replace all spectator fields by this matrix. The non-wrapping contributions to $V_{2 R}^{(1)}$ would then vanish automatically when used to build the 2-point function of these modified operators $\emptyset e 2 R$.

In order to find such a matrix, we parameterize $\psi, \hat{\psi}$ as $\psi=\hat{\psi}=\psi_{0} T^{0}+$ $\psi_{a} T^{a}, a=1, \ldots, N^{2}-1$ with respect to the decomposition of $U(N)$ into a $U(1)$ part and an $S U(N)$ part. The coefficients $\psi_{0}$ and $\psi_{a}$ are complex. A non 
traceless $\psi$ requires that $\psi_{0} \neq 0$. The condition that $\operatorname{tr}\left(\psi^{2}\right)=0$ amounts to the 'light-cone' condition

$$
\psi_{a} \psi^{a}+\psi_{0}^{2}=0,
$$

where we have used $(\mathbb{B} .1)$ and $(\mathbb{B} .2)$. The vanishing of the traces that contain higher powers of $\psi$ is greatly simplified by the recursive use of the relations found up to the step before. For example, evaluation of $\operatorname{tr}\left(\psi^{3}\right)$ and use of (6.1) gives

$$
\psi_{a} \psi_{b} \psi_{c} \operatorname{tr}\left(T^{a} T^{b} T^{c}\right)-\frac{2}{\sqrt{N}} \psi_{0}^{3}=0
$$

After a little thought, one realizes that the general form of this equation at order $n$ is

$$
\psi_{a_{1}} \ldots \psi_{a_{n}} \operatorname{tr}\left(T^{a_{1}} \ldots T^{a_{n}}\right)+\frac{c_{n}}{\sqrt{N}^{n-2}} \psi_{0}^{n}=0
$$

where the $c_{n}$ are determined by recursion as

$$
c_{n}=1-\left(\begin{array}{l}
n \\
2
\end{array}\right)-\sum_{m=3}^{n-1}\left(\begin{array}{l}
n \\
m
\end{array}\right) c_{m}
$$

for $n \geq 3$ (for $n=3$ the sum has to be considered empty). This recursion is solved by

$$
c_{n}=(-1)^{n}(n-1),
$$

as can be easily verified using properties of binomial sums. The constraint on $\psi$ is given by (6.1) combined with

$$
\psi_{a_{1}} \ldots \psi_{a_{n}} \operatorname{tr}\left(T^{a_{1}} \ldots T^{a_{n}}\right)+\frac{(-1)^{n}}{\sqrt{N}^{n-2}}(n-1) \psi_{0}^{n}=0
$$

for $n=3, \ldots, n_{0}$ up to a desired order, which coincides with the highest possible power of $\psi$ within a single trace, obviously given by $n_{0}=2 R$.

The choice $\psi_{0}=\frac{1}{\sqrt{N}}$ normalizes the trace $(\psi)=1$, such that the additional matrices within the traces do not change the prefactor for the planar wrapping interactions. The conditions (6.1) and (6.6) then read

$$
\psi_{a} \psi^{a}=-\frac{1}{N}, \quad \psi_{a_{1}} \ldots \psi_{a_{n}} \operatorname{tr}\left(T^{a_{1}} \ldots T^{a_{n}}\right)+\frac{(-1)^{n}}{N^{n-1}}(n-1)=0 .
$$

The important question to answer is whether this set of conditions has a solution in general. This involves determining the components of the tensors 
$k^{a_{1} \ldots a_{n}}=\operatorname{tr}\left(T^{\left(a_{1}\right.} \ldots T^{\left.a_{n}\right)}\right)$ appearing in (6.6), where the parentheses indicate complete symmetrization with unit weight (i.e. summing over all permutations, and dividing by $n$ !). These objects are a family of symmetric invariant tensors of $S U(N)$ [39. Not all of them are independent. For $n \geq N+1 k^{a_{1} \ldots a_{n}}$ can be expressed in terms of the lower order ones. Instead of working with these objects, we have tried to test whether it is possible to find any matrix $M$ with a non-vanishing trace, such that $M^{2}, M^{3}$ and $M^{4}$ have vanishing trace. We were able to find a diagonal matrix of rank 12 with pairs of complex conjugated eigenvalues $\lambda_{i}, \bar{\lambda}_{i}$ of unit length given by

$$
\lambda_{1,2}=-1, \quad \lambda_{3}=\frac{1+i \sqrt{3}}{2}, \quad \lambda_{4,5,6}=\frac{-1+i \sqrt{3}}{2} .
$$

\section{Conclusions}

In this paper we have studied the genus expansion of the 2-point function of single-trace operators containing $R$ elementary fields, and of one of its building blocks which is the Green function with $2 R$ external legs. We have shown that the two genus expansions do not coincide. The contributions to the Green function of a fixed genus $h$ lead to genus $h-2 \leq H \leq h$ contributions to the 2 -point function. We have discussed the two origins for this effect. Firstly, a reduction of the genus is caused by the invariance of the trace of the composite operators under cyclic permutations. Secondly, a reduction of the genus is caused by the fact that certain crossings between field lines in some contributions to the Green function can be resolved when it is used for the construction of the 2-point function. In these wrapping contributions some field lines occupy a special (wrapping) path, present in case of the 2-point function, thereby avoiding some crossings that would require adding a handle. These wrapping contributions play a role whenever the range of an interaction becomes larger than the length of the object it acts on. In the context of the AdS/CFT correspondence it is believed that they can explain an observed mismatch between the energies of classical strings and the anomalous dimensions eigenvalues of the corresponding operators starting at three loops.

To avoid the additional genus changes caused by the cyclic symmetry of the local operators, we have introduced equivalence classes for the diagrams of the Green function. Diagrams that differ only by cyclic permutations within the ingoing and outgoing legs are identified, and for the construction of the 2-point function only one diagram with minimal genus $h$ is taken from each equivalence 
class. As a result of the analysis we have found that all genus $H$ contributions to the 2-point function of composite single-trace operators are obtained from these genus $h=H$ and $h=H+1$ contributions to the Green function.

In our opinion this observation should be of particular importance. At a given sufficiently high order in the coupling constant, one can divide the contributions to the 2-point function and to the Green function into two classes. One class contains those contributions which are universal, i.e. they have to be used in the construction of the 2-point function of operators with a larger number of legs. The second class contains those contributions which are non universal. Its elements change whenever the number of legs of the operators is changed. However, our above given result shows that even these non universal contributions are contained within a universal quantity, the Green function. Thus, the non universality of the elements within the second class can be understood as a non universality of the projection operation used to obtain these elements. A better understanding of the projection operation might therefore provide a way to obtain the wrapping contributions without the need to implement a selection process on each diagram separately.

As a first step into this direction, we have worked out a technique, that allows one to identify the wrapping contributions in the planar $(H=0)$ case, which is of particular interest in the context of the AdS/CFT correspondence. The technique is based on adding pairs of spectator fields and their pairwise connections to the diagrams of the 2-point function. The planar wrapping contributions are then identified as the only contributions in which, after contracting all the other fields, the trace over each matrix-valued spectator field is taken separately. A particular choice for the components of the spectator fields should therefore project out the planar wrapping contributions automatically. We have determined the equations which such a matrix must fulfill and we have presented a simple example to demonstrate that the problem itself should have a solution.

Clearly, our analysis is only a first step to a complete understanding of the wrapping interactions. As a next step one could try to restrict the freedom in our proposed general vertex that should describe wrapping interactions in the traceless $S U(2)$ subsector. It would be interesting to discuss whether such an interaction term can be compatible with integrability. 


\section{Acknowledgements}

We would like to thank B. Eden, J. Plefka and E. Sokatchev for deep and enlightening discussions. The work of C. S. is supported by DFG (Deutsche Forschungsgemeinschaft) within 'Graduiertenkolleg 271' and within project ER 301/1-4. The work of A. T. is supported by DFG within the 'Schwerpunktprogramm Stringtheorie 1096'. 


\section{A Counting rules for Feynman diagrams}

We use the quantities

$$
\begin{aligned}
K & =\text { order of the diagram } g^{K} \\
E & =\text { number of external lines } \\
P & =\text { number of propagators } \\
V_{i}^{k} & =\text { number of vertices of order } g^{k} \text { with } i \text { legs } \\
V & =\text { number of vertices } \\
L & =\text { number of loops } \\
C & =\text { number of connected pieces }
\end{aligned}
$$

that classify a generic Feynman diagram. The following relations hold

$$
K=\sum_{i, k} k V_{i}^{k}, V=\sum_{i, k} V_{i}^{k}, E=\sum_{i, k} i V_{i}^{k}-2 P, P=V+L-C .
$$

The equation for $E$ can be cast into the form

$$
E=\sum_{i, k}(i-2) V_{i}^{k}-2(L-C)
$$

It simplifies if the $V_{i}^{k}$ obey

$$
V_{i}^{k}=V_{k+2}^{k} \delta_{i, k+2}
$$

i.e. from the coupling constant $g$ of the three point vertex all coupling constants of the higher point vertices are uniquely determined by adding one power in $g$ for each additional leg. For example, in YM theories one only has the cubic and quartic vertices, and therefore respectively $V_{3}^{1}$ and $V_{4}^{2}$ are different from zero. With (A.4) one finds that (A.2) simplifies to

$$
K=\sum_{k} k V_{k+2}^{k}, V=\sum_{k} V_{k+2}^{k}, E=K-2(L-C), P=V+L-C .
$$

Consider now the special case of a 2-point function $\left(\varnothing 1 R, V_{2 R}, \varnothing 2 R\right)$ of two composite operators $\varnothing e R$, where all vertices in $V_{2 R}$ are of the form (A.4). One can regard the operators as two vertices that do not contribute to the order in the coupling constant and that have $R$ legs, i.e. $V_{R}^{0}=2$. Furthermore, one has 
$E=0$ since all legs of $\varnothing 1 R$ are contracted with the external legs of $V_{2 R}$. From the equation for $E$ in $(\mathrm{A} .2$ ) one then finds in this case

$$
K=2(L-C-R+2) .
$$

One can now determine the minimum order $K$ for a planar wrapping diagram to appear. For this purpose, one has to estimate the minimum number of $L$. The simplest non-interacting case in Subsection 4.1 with $K=0, C=1$ inserted into (A.6) gives $L=R-1$. In this case one can add $R$ spectators to the diagram without making it non-planar by crossing any other lines. Therefore, each of the $R-1$ loops has to be divided into at least 2-loops by a line that crosses a spectator line. Furthermore, one has to add at least one line that runs along the wrapping path, increasing the loop number by at least one. The number of loops in a wrapping diagram thus fulfills $L \geq 2 R-1$. Clearly $C=1$, and (A.6) then leads to a lower bound for $K$ that becomes

$$
K \geq 2 R \text {. }
$$

The structure of a generic planar wrapping diagram is arbitrarily complicated. In particular, the interaction part that fills the wrapping path can itself be an arbitrary planar diagram, see Fig. 9. However, up to a certain order $K$ in the coupling constant, all wrapping diagrams can be obtained from planar non-wrapping connected diagrams of maximum interaction length by adding a single line along the wrapping path.

For a given wrapping diagram one can use the cyclic symmetry to minimize the number of lines that run along the wrapping path. One only has to guarantee that the order $K$ in the coupling constant is sufficiently low such that at least one spectator line can be added that only crosses a single line of the planar diagram. This means all the other spectator lines in this case are allowed to cross at most two other lines. If one of them crosses more than two other lines, one automatically finds a diagram at the same order where none of the spectator lines crosses only a single line. This is due to the fact that one could replace the third crossed line by a line that generates a second crossing of the spectator line that previously crossed only one other line. Since each of the lines that connect neighboured fields of the operators in a given diagram effectively contributes with a factor $g^{2}$, the order $K$ for which all wrapping diagrams can be obtained by adding a single line to the planar non-wrapping connected diagrams of maximum interaction length has to fulfill

$$
K \leq 2(2(R-1)+1)=2(2 R-1) .
$$




\section{B Rules for $U(N)$ and $S U(N)$}

Let $\left(T^{a}\right)^{i}{ }_{j}, a=a_{0}, \ldots N^{2}-1, i, j=1 \ldots N$ be the $N \times N$ representation matrices for the Lie algebras of $U(N)$ (with $a_{0}=0$ ) and $S U(N)$ (with $a_{0}=1$ ). They are chosen such that they fulfill

$$
\left[T^{a}, T^{b}\right]=i f^{a b c} T^{c}, \quad \operatorname{tr}\left(T^{a} T^{b}\right)=\delta^{a b},
$$

where the trace tr is taken over the fundamental indices $i, j$ respectively. In particular, the $U(1)$ generator is given by

$$
T^{0}=\frac{1}{\sqrt{N}} \mathbb{1}
$$

The representation matrices then fulfill the relation

$$
\sum_{a=a_{0}}^{N^{2}-1}\left(T^{a}\right)^{i}{ }_{j}\left(T^{a}\right)^{k}{ }_{l}=\delta_{l}^{i} \delta_{j}^{k}-\frac{a_{0}}{N} \delta_{j}^{i} \delta_{l}^{k},
$$

where as above $a_{0}=0$ and $a_{0}=1$ for $U(N)$ and $S U(N)$ respectively. With the help of the above relation it is straigthforward to obtain the fusion and fission rules for the traces

$$
\begin{aligned}
\operatorname{tr}\left(T^{a} A\right) \operatorname{tr}\left(T^{a} B\right) & =\operatorname{tr}(A B)-\frac{a_{0}}{N} \operatorname{tr}(A) \operatorname{tr}(B), \\
\operatorname{tr}\left(T^{a} A T^{a} B\right) & =\operatorname{tr}(A) \operatorname{tr}(B)-\frac{a_{0}}{N} \operatorname{tr}(A B) .
\end{aligned}
$$

In the main text we use an abbreviated notation where the traces are simply represented by parentheses and indices are no longer written as superscripts. The above rules then read

$$
\begin{aligned}
(a A)(a B) & =(A B)-\frac{a_{0}}{N}(A)(B), \\
(a A a B) & =(A)(B)-\frac{a_{0}}{N}(A B) .
\end{aligned}
$$




\section{References}

[1] J. M. Maldacena, "The large $N$ limit of superconformal field theories and supergravity," Adv. Theor. Math. Phys. 2 (1998) 231-252, hep-th/9711200

[2] S. S. Gubser, I. R. Klebanov, and A. M. Polyakov, "A semi-classical limit of the gauge/string correspondence," Nucl. Phys. B636 (2002) 99-114, hep-th/0204051.

[3] S. Frolov and A. A. Tseytlin, "Semiclassical quantization of rotating superstring in $\mathrm{AdS}_{5} \times \mathrm{S}^{5}$," JHEP 06 (2002) 007, hep-th/0204226.

[4] A. A. Tseytlin, "Spinning strings and AdS/CFT duality," hep-th/0311139

[5] S. A. Frolov, I. Y. Park, and A. A. Tseytlin, "On one-loop correction to energy of spinning strings in S ${ }^{5}$," Phys. Rev. D71 (2005) 026006, hep-th/0408187

[6] G. Arutyunov, S. Frolov, J. Russo, and A. A. Tseytlin, "Spinning strings in $\mathrm{AdS}_{5} \times \mathrm{S}^{5}$ and integrable systems," Nucl. Phys. B671 (2003) 3-50, hep-th/0307191

[7] G. Arutyunov, J. Russo, and A. A. Tseytlin, "Spinning strings in $\mathrm{AdS}_{5} \times \mathrm{S}^{5}$ : New integrable system relations," Phys. Rev. D69 (2004) 086009, hep-th/0311004

[8] L. F. Alday, G. Arutyunov, and A. A. Tseytlin, "On integrability of classical superstrings in $\mathrm{AdS}_{5} \times \mathrm{S}^{5}, "$ hep-th/0502240

[9] I. Swanson, "On the integrability of string theory in $\mathrm{AdS}_{5} \times \mathrm{S}^{5}$," hep-th/0405172

[10] I. Swanson, "Quantum string integrability and AdS/CFT," Nucl. Phys. B709 (2005) 443-464, hep-th/0410282

[11] V. A. Kazakov, A. Marshakov, J. A. Minahan, and K. Zarembo, "Classical / quantum integrability in AdS/CFT," JHEP 05 (2004) 024, hep-th/0402207.

[12] G. Arutyunov and S. Frolov, "Integrable Hamiltonian for classical strings on $\mathrm{AdS}_{5} \times \mathrm{S}^{5}, " J H E P 02$ (2005) 059, hep-th/0411089

[13] N. Beisert, C. Kristjansen, and M. Staudacher, "The dilatation operator of $\mathcal{N}=4$ super Yang-Mills theory," Nucl. Phys. B664 (2003) 131-184, hep-th/0303060.

[14] J. C. Plefka, "Lectures on the plane-wave string / gauge theory duality," Fortsch. Phys. 52 (2004) 264-301, hep-th/0307101.

[15] N. Beisert, "The dilatation operator of $\mathcal{N}=4$ super Yang-Mills theory and integrability," Phys. Rept. 405 (2005) 1-202, hep-th/0407277

[16] J. A. Minahan and K. Zarembo, "The Bethe-ansatz for $\mathcal{N}=4$ super Yang-Mills," JHEP 03 (2003) 013, hep-th/0212208

[17] H. Bethe, "On the theory of metals. 1. Eigenvalues and eigenfunctions for the linear atomic chain," Z. Phys. 71 (1931) 205-226.

[18] L. D. Faddeev, "How Algebraic Bethe Ansatz works for integrable model," hep-th/9605187 
[19] N. Beisert, S. Frolov, M. Staudacher, and A. A. Tseytlin, "Precision spectroscopy of AdS/CFT," JHEP 10 (2003) 037, hep-th/0308117.

[20] D. Berenstein, J. M. Maldacena, and H. Nastase, "Strings in flat space and pp waves from $\mathcal{N}=4$ super Yang-Mills," JHEP 04 (2002) 013, hep-th/0202021

[21] R. Penrose, "A remarkable property of plane waves in general relativity," Rev. Mod. Phys. 37 (1965) 215-220.

[22] R. Güven, "Plane wave limits and T-duality," Phys. Lett. B482 (2000) 255-263, hep-th/0005061

[23] M. Blau, J. Figueroa-O'Farrill, C. Hull, and G. Papadopoulos, "A new maximally supersymmetric background of II B superstring theory," JHEP 01 (2002) 047, hep-th/0110242

[24] M. Blau, J. Figueroa-O'Farrill, and G. Papadopoulos, "Penrose limits, supergravity and brane dynamics," Class. Quant. Grav. 19 (2002) 4753, hep-th/0202111

[25] J. Callan, Curtis G. et al., "Quantizing string theory in $\mathrm{AdS}_{5} \times \mathrm{S}^{5}$ : Beyond the pp-wave," Nucl. Phys. B673 (2003) 3-40, hep-th/0307032

[26] J. Callan, Curtis G., T. McLoughlin, and I. Swanson, "Holography beyond the Penrose limit," Nucl. Phys. B694 (2004) 115-169, hep-th/0404007

[27] J. Callan, Curtis G., T. McLoughlin, and I. Swanson, "Higher impurity AdS/CFT correspondence in the near-BMN limit," Nucl. Phys. B700 (2004) 271-312, hep-th/0405153

[28] T. McLoughlin and I. Swanson, "N-impurity superstring spectra near the pp-wave limit," Nucl. Phys. B702 (2004) 86-108, hep-th/0407240

[29] I. Y. Park, A. Tirziu, and A. A. Tseytlin, "Spinning strings in $\mathrm{AdS}_{5} \times \mathrm{S}^{5}$ : One-loop correction to energy in $S L(2)$ sector," JHEP 03 (2005) 013, hep-th/0501203

[30] M. Lubcke and K. Zarembo, "Finite-size corrections to anomalous dimensions in $\mathcal{N}=4$ SYM theory," JHEP 05 (2004) 049, hep-th/0405055

[31] V. A. Kazakov and K. Zarembo, "Classical / quantum integrability in non-compact sector of AdS/CFT," JHEP 10 (2004) 060, hep-th/0410105

[32] N. Beisert, A. A. Tseytlin, and K. Zarembo, "Matching quantum strings to quantum spins: One-loop vs. finite-size corrections," Nucl. Phys. B715 (2005) 190-210, hep-th/0502173

[33] D. Serban and M. Staudacher, "Planar $\mathcal{N}=4$ gauge theory and the Inozemtsev long range spin chain," JHEP 06 (2004) 001, hep-th/0401057

[34] N. Beisert, V. Dippel, and M. Staudacher, "A novel long range spin chain and planar $\mathcal{N}=4$ super Yang-Mills," JHEP 07 (2004) 075, hep-th/0405001

[35] T. Fischbacher, T. Klose, and J. Plefka, "Planar plane-wave matrix theory at the four loop order: Integrability without BMN scaling," JHEP 02 (2005) 039, hep-th/0412331 
[36] G. 't Hooft, "A planar diagram theory for the strong interactions," Nucl. Phys. B72 (1974) 461.

[37] K. G. Chetyrkin, A. L. Kataev, and F. V. Tkachov, "New approach to evaluation of multiloop Feynman integrals: the Gegenbauer Polynomial $x$ space technique," Nucl. Phys. B174 (1980) 345-377.

[38] D. I. Kazakov and A. V. Kotikov, "The method of uniqueness: multiloop calculations in QCD," Theor. Math. Phys. 73 (1988) 1264.

[39] J. A. de Azcárraga, A. J. Macfarlane, A. J. Mountain, and J. C. Pérez Bueno, "Invariant tensors for simple groups," Nucl. Phys. B510 (1998) 657-687, physics/9706006 